\title{
Anatomical and physiological basis of continuous spike-wave of sleep syndrome after early thalamic lesions
}

\author{
Alberto Leal ${ }^{\mathrm{a}, *}$, Eulália Calado ${ }^{\mathrm{b}}$, José P. Vieira ${ }^{\mathrm{b}}$, Carla Mendonça ${ }^{\mathrm{c}}$, José C. Ferreira ${ }^{\mathrm{d}}$, Hugo Ferreira ${ }^{\mathrm{e}}$, \\ Daniel Carvalho ${ }^{\mathrm{b}}$, Fátima Furtado ${ }^{\mathrm{f}}$, Roseli Gomes ${ }^{\mathrm{g}}$, José P. Monteiro ${ }^{\mathrm{h}}$ \\ a Department of Neurophysiology, Centro Hospitalar Psiquiátrico de Lisboa, Avenida do Brasil 53, 1749.002 Lisbon, Portugal \\ b Centro Hospitalar Lisboa Central, Pediatric Neurology Department, Lisbon, Portugal \\ c Centro Hospitalar do Algarve, Pediatrics Department, Faro, Portugal \\ d Centro Hospitalar Lisboa Ocidental, Pediatrics Department, Lisbon, Portugal \\ e Centro Hospitalar Lisboa Central, Neurology Department, Lisbon, Portugal \\ ${ }^{\mathrm{f}}$ Unidade Local Saúde Baixo Alentejo, Pediatrics Department, Beja, Portugal \\ ' Unidade Local Saúde de Matosinhos, Pediatrics Department, Matosinhos, Portugal \\ ${ }^{\text {h }}$ Hospital Garcia de Orta, Pediatric Neurology Department, Almada, Portugal
}

\section{A R T I C L E I N F O}

\section{Article history:}

Received 16 March 2017

Revised 18 August 2017

Accepted 19 August 2017

Available online 11 November 2017

\section{Keywords:}

CSWS syndrome

Thalamic lesion

Epilepsy

Augmenting response

\begin{abstract}
A B S T R A C T
Objective: Early neonatal thalamic lesions account for about $14 \%$ of continuous spike-wave of sleep (CSWS) syndrome, representing the most common etiology in this epileptic encephalopathy in children, and promise useful insights into the pathophysiology of the disease.

Methods: We describe nine patients with unilateral neonatal thalamic lesions which progressed to CSWS. Longitudinal whole-night and high-density electroencephalograms (EEGs) were performed, as well as detailed imaging and clinical evaluation. Visual evoked potentials were used to probe cortical excitability.

Results: Thalamic volume loss ranged from $19 \%$ to $94 \%$, predominantly on medial and dorsal nuclei and sparing the ventral thalamus. Lesions produced white matter loss and ventricle enlargement on the same hemisphere, which in four patients was associated with selective loss of thalamic-cortical fibers. Cortical thickness quantification failed to reveal hemispheric asymmetries. Impact on EEG rhythms was mild, with a volume-loss-related decrease in alpha power and preservation of sleep spindles. The sleep continuous spiking was lateralized to the hemisphere with the lesion. Visual cortex stimulation in five patients with posterior cortex spiking revealed an abnormal frequency-dependent excitability at $10-20 \mathrm{~Hz}$ on the side of the lesion.

Significance: Unilateral selective thalamic-cortical disconnection is a common feature in our patients and is associated with both a focal pattern of CSWS and a pathological type of frequency-dependent excitability (peak: $10-20 \mathrm{~Hz}$ ). We propose that this excitability represents an abnormal synaptic plasticity previously described as the augmenting response. This synaptic plasticity has been described as absent in the corticocortical interactions in healthy experimental animals, emerging after ablation of the thalamus and producing a frequencydependent potentiation with a peak at 10-20 Hz. Because this response is potentiated by sleep states of reduced brainstem activation and by appropriate stimulating rhythms, such as sleep spindles, the simultaneous occurrence of these two factors in nonrapid-eye-movement sleep is proposed as an explanation for CSWS in our patients.
\end{abstract}

(C) 2017 Elsevier Inc. All rights reserved.

\section{Introduction}

The syndrome of continuous spike-wave of sleep (CSWS) is an important treatable epileptic encephalopathy with peculiar features that

Abbreviations: CSWS, continuous spike-wave of sleep; VPS, ventricle-peritoneal shunt; HR-EEG, high-resolution electroencephalogram; DWI, diffusion weighted imaging; Thal-Bundle, thalamic bundle; SSVEPs, steady-state visual evoked potentials; SNR, signalnoise ratio; sLORETA, standard low-resolution tomography.

* Corresponding author.

E-mail address: albertoleal@chpl.min-saude.pt (A. Leal). set it apart from the seizure-related morbidity associated with other types of epilepsies [1]. Retrospectively, the first examples clearly identifiable as CSWS were reported [2] in six children. The peculiar appearance of continuous spiking with sleep onset, the fast disappearance with both rapid eye movement (REM) and waking, and the persistent occurrence "night after night" for years were all described in this early report. More recently, the syndrome was reclassified as an age-related and self-limited childhood encephalopathy [3]. An incidence of neuroradiological abnormalities in between $33 \%$ and $60 \%$ of cases with CSWS have been reported in several series [4], with lesions dominated by unilateral or diffuse atrophy, but cases of porencephaly, pachygyria, 
cortical developmental disorders, polymicrogyria, and hydrocephalus have also been reported. A more recent list [5] adds yet other lesions but, most importantly, highlights the importance of early vascular thalamic lesions.

The connection between early thalamic lesions and CSWS was first suggested in case reports [6-8]. A large series $(N=32)$ of patients with early thalamic lesions strengthened the case for this connection [9] by demonstrating that $37.5 \%$ of patients with early thalamic lesions presented the syndrome years later. An enlarged series from the same group with $N=60$ patients [10] and a more careful prospective study of the new cases revealed that patients with thalamic lesions involving predominantly mediodorsal nuclei had a higher probability of evolving to CSWS. Kersbergen et al. [11] followed prospectively 14 survivors of early thalamic hemorrhages and found CSWS-like (spike index $>50 \%$ ) in 5 , sleep-induced epileptiform activity in 2, and focal epilepsy in another 2 . The young age of some of the patients, below the typical age of onset of CSWS, suggests that these numbers are lower estimates, and yet they support a relationship between the two pathologies. The relative importance of this etiology in the common CSWS of the general population was addressed in a group of $N=100$ children [5] with a CSWS-like clinical presentation and spike index higher than $50 \%$ in which $14 \%$ had evidence for thalamic lesions, the single most important etiology.

In the present study, we characterize the neurophysiological features of CSWS in a group of patients with neonatal thalamic lesions and propose an anatomical and physiological model for the origin of the associated sleep-related continuous spiking.

\section{Methods}

\subsection{Clinical data}

Clinical data for the nine patients in the present series are shown in Table 1. Patients were selected from a population of children with CSWS evaluated at the clinical neurophysiology laboratory of the first author in the time span of 15 years, with the additional requirement that a well-defined thalamic lesion was identified in the magnetic resonance imaging (MRI) study. Cases with diffuse encephalopathy, hypoxicischemic etiology, or structural lesions extending beyond the thalamus were excluded.

In all patients, the lesion was unilateral, and seven out of nine had a clear history of neonatal thalamic hemorrhage, with an acute severe brain disease which in two cases required the placement of a ventricle-peritoneal shunt (VPS) (Fig. 4B). Of the patients with no history of thalamic hemorrhage, one had no pathological events preceding the epilepsy onset and consequent MRI diagnosis (patient 2), whereas in the other case (patient 5), preeclampsia was diagnosed in the late stages of pregnancy and the posterior MRI showed thalamic deposition of hemosiderin supporting a previous hemorrhagic event (Supplementary Fig. 1).

Most patients suffering thalamic hemorrhage recovered surprisingly well, and four out of seven met the early motor and cognitive developmental milestones, (Table 1). In three patients, this development was delayed, which in two cases was partially attributed to surgical complications associated with the management of the hydrocephalus.

In most patients, the later onset of CSWS was associated with significant deterioration of behavioral and cognitive capabilities (Table 1).

\subsection{Electroencephalogram (EEG) acquisition and processing}

All patients were submitted to repeated whole-night EEG recordings using the 10-20 system plus subtemporal electrodes (F9/10 and P9/10) and quantification of the Spike Index (SI) using a modification of the method of Larsson et al. [12], with determination of the ratio of 3-s epochs with spikes and without spikes in an integration time of $30 \mathrm{~s}$ (instead of the 10-min period in the original method), running through the whole EEG trace. With this modification, we could perform a quantification of the SI for each sleep stage evaluation. Patient P8 had a whole-night recording only after CSWS resolution and clearing of the EEG spikes, so an SI quantification was not possible.

A high-resolution electroencephalogram (HR-EEG) was performed in eight of the nine patients. This consisted of 1- to 2-h recordings using a cap (EasyCap Inc) with 82 electrodes, corresponding to the full 10-10 system plus electrodes F11/12, FT11/12, TP11/12, and P11/12, and electrocardiogram. A digital EEG amplifier was used (SynAmps2, Compumedics-Neuroscan), with sintered $\mathrm{AgCl}$ ring electrodes, a sampling rate of $1000 \mathrm{~Hz}$, and filters DC $70 \mathrm{~Hz}$. The first part of the recording included resting-state and visual evoked potentials, whereas the second part included a 30-min nap. Localization of the spike activity of CSWS was performed using a realistic boundary element model (BEM), obtained from segmentation of the anatomical T1 sequence for each patient. Scalp maps of the average and Laplacian representative spike at half-peak and peak amplitude were computed along with source analysis using the cortical standard low-resolution tomography (sLORETA) model implemented in the CURRY6.0 software (CompumedicsNeuroscan). In one patient, we could not obtain the Laplacian because he did not cooperate to obtain an acceptable HR-EEG.

The posterior alpha and mu spectral data were obtained from the HR-EEG at rest with eyes closed and eyes open, respectively. The average spectra of several (15 to 20) 1-s epochs for each channel (applying a Hanning window and fast Fourier transform [FFT]) were computed and used to draw a scalp representation map of the power distribution. The interhemispheric ratio was computed from the power in electrodes $\mathrm{C} 3 / 4$ for $\mathrm{Mu}$ and the average power of electrodes P7/8, CP3/4, and 01/2 for the posterior alpha. Spindles were manually located in the $\mathrm{Cz}$ channel of whole-night EEG recordings, and 30-50 1-s epochs were obtained and used to compute the average spectra for each channel. With these spectra, we compared hemispheres (using the C3-F7 and C4-F8 derivations) and obtained a global map of power distribution at the peak of spindles. The asymmetry index $(\mathrm{AI})$ was computed as $\mathrm{AI}=(P-N) /$ $(P+N)^{*} 100$, where $P$ and $N$ are the power in pathological and normal hemispheres [13].

The correlation between SI and the sleep stage was obtained by manually scoring the whole-night EEG recordings using EEG derivations from the nonlesioned hemisphere. For each sleep stage, we computed the average SI for the whole night.

\subsection{Imaging of and volumetry data}

In all patients, an anatomical whole-brain MRI was obtained, which allowed manual volume quantification of the whole thalamus, using the methodology described in Power et al. [14] and pulvinar using the guidelines of Byne et al. [15]. Because lesions were always unilateral, volumes were normalized to the healthy hemisphere. In six patients, automatic quantification of the cortical thickness using the software FreeSurfer was performed. In order to compare diverse cortical areas, the cortical reconstruction of each hemisphere was automatically parceled into the 74 areas of the Destrieux atlas, and the average cortical thickness of each area was compared between hemispheres.

In four patients, diffusion-weighted imaging (DWI) was obtained using the following parameters: spin-echo echo-planar imaging sequence with $b=1000 \mathrm{~s} / \mathrm{mm}^{2}, 25$ directions, 1 non-DWI image, resolution $=4.0 \times 4.0 \times 4.0 \mathrm{~mm}^{3}, 36$ slices with no gap, and echo time/repetition time of $89.7 / 8475 \mathrm{~ms}$. Deterministic tractography quantification of the structural connectivity between the thalamus and temporal lobes and also of the pyramidal pathway was performed (Fig. 5A) after standard preprocessing steps, including Eddy current distortion, with the freely available software tool DiffusionKit (http://diffusionkit. readthedocs.io) [16]. After fiber reconstruction using the manually segmented thalamus as seed and excluding vertically and posteriorly projecting bundles using appropriate exclusion planes (Fig. 5A, left), we identified the two most important bundles projecting to the 
Table 1

\begin{tabular}{|c|c|c|c|c|c|c|c|c|c|}
\hline & P1 & $\mathrm{P} 2$ & P3 & P4 & P5 & P6 & P7 & P8 & P9 \\
\hline Age & $17 \mathrm{y}$ & $23 \mathrm{y}$ & $12 \mathrm{y}$ & $13 \mathrm{y}$ & $9 y$ & $8 \mathrm{y}$ & $7 y$ & $15 \mathrm{y}$ & $13 \mathrm{y}$ \\
\hline Sex & Male & Male & Male & Male & Female & Female & Female & Female & Female \\
\hline Gestation & Uneventful, ft & Uneventful, $\mathrm{ft}$ & Uneventful, ft & Uneventful, ft & Preeclampsia, ft & Uneventful, $\mathrm{ft}$ & Uneventful, ft & Uneventful, ft & Uneventful, ft \\
\hline \multirow[t]{2}{*}{ Delivery } & Pelvic & Pelvic & $\begin{array}{l}\text { Cesarean (placental } \\
\text { displacement) }\end{array}$ & Pelvic & Cesarean & $\begin{array}{l}\text { Pelvic, forceps } \\
\text { (neonatal resuscitation) }\end{array}$ & Pelvic & Pelvic & Pelvic \\
\hline & APGAR 8/10 & & APGAR $8 / 9$ & APGAR 9/10 & APGAR 9/10 & APGAR 5/7 & & & APGAR 9/10 \\
\hline \multicolumn{10}{|l|}{ Neonatal period } \\
\hline Thalamic hemorrhage & Not detected & (Left, day 33) & (Left thal, day 9) & (Right thal, day 18) & Not detected & (Right thal, day 3) & (Right thal, day 2) & (Left thal, day 22) & (Left thal, day 2) \\
\hline Clinical manifestations & None & Seizure + cardiac arrest & Seizure & seizures, vomiting & None & Seizures & Seizures & & Seizures \\
\hline Comorbidities & None & Ventricular bleeding & $\begin{array}{l}\text { Sepsis, venous thromb, } \\
\text { ventricular bleeding }\end{array}$ & Ventricular bleeding, & None & $\begin{array}{l}\text { Ventricular bleeding, } \\
\text { VPS, cleft palate }\end{array}$ & $\begin{array}{l}\text { Ventricular } \\
\text { bleeding }\end{array}$ & $\begin{array}{l}\text { Ventricular bleeding, } \\
\text { hydrocephalus, VPS }\end{array}$ & $\begin{array}{l}\text { Ventricular bleeding, } \\
\text { hydrocephalus }\end{array}$ \\
\hline \multicolumn{10}{|l|}{ Develop milestones } \\
\hline Independent walking & Normal & Normal & $\begin{array}{l}1 \text { y } 6 \mathrm{mo} \\
\text { (mild right foot paresis) }\end{array}$ & Normal & Normal & $\begin{array}{l}3 \mathrm{y} \\
\text { (mild left hemiparesis) }\end{array}$ & Normal & $\begin{array}{l}2 \mathrm{y} \\
\text { (delayed acquisition) }\end{array}$ & Normal \\
\hline Speech acquisition & Normal & Normal & Normal & Normal & Normal & $\begin{array}{l}5 \mathrm{y} \\
\text { (delayed acquisition) }\end{array}$ & Normal & $\begin{array}{l}4 \mathrm{y} \\
\text { (delayed acquisition) }\end{array}$ & Normal \\
\hline \multicolumn{10}{|l|}{ Seizures } \\
\hline onset & $3 y$ & $3 y$ & 2 y $5 \mathrm{mo}$ & $7 \mathrm{mo}$ & $2 \mathrm{y}$ & IS (3 mo); CPS (4 y) & $19 \mathrm{mo}$ & $6 y$ & $5 \mathrm{y}$ \\
\hline Frequency & 1 each 3 mo & Daily & Daily & & & Weekly & Monthly & $1-2 / y$ & $1-2 / y$ \\
\hline Clinical features & CPS & CPS; PMS; right visual & CPS & CPS & Left PMS & CPS & Left PMS; CPS & GTC seizures & CPS \\
\hline Seizure-free & From age $12 \mathrm{y}$ & From age $10 \mathrm{y}$ & Sporadic seizures & From age $7 \mathrm{y}$ & From age $9 \mathrm{y}$ & Sporadic seizures & From age $6 \mathrm{y}$ & From age $9 y$ & From age $8 \mathrm{y}$ \\
\hline \multicolumn{10}{|l|}{ CSWS diagnosis } \\
\hline Behavioral regression & (Language and $\mathrm{S} / \mathrm{T}$ ) & (Scholar performance) & (Language and $\mathrm{S} / \mathrm{T}$ ) & (Language and motor) & $\begin{array}{l}\text { (Scholar } \\
\text { performance) }\end{array}$ & (Not apparent) & (Not apparent) & (Not apparent) & $\begin{array}{l}\text { (Scholar } \\
\text { performance) }\end{array}$ \\
\hline \multicolumn{10}{|l|}{ Cognition } \\
\hline Daily activities & Average low & Good performance & Developmental delay & Developmental delay & Average low & Developmental delay & Good & Developmental delay & Good \\
\hline School performance & Learning disability & Average low & Severe learning disab & Severe learning disab & Average low & Severe learning disab & Above average & Severe learning disab & Average \\
\hline Overall & IQ 70 (9 y) & Normal & Griffiths 87 (4 y 9 mo) & Severe delay & IQ 80 (7 y) & Severe delay & $\begin{array}{l}\text { Griffiths } 91 \\
(2 \text { y } 7 \text { mo })\end{array}$ & IQ 57 (14 y) & $\begin{array}{l}\text { IQ70 (8 y); IQ } 79 \\
(10 y)\end{array}$ \\
\hline
\end{tabular}

VPS, ventricle-peritoneal shunt; ft, full term; IS, infantile spams; CPS, complex partial seizures; PMS, partial motor seizures; GTC, generalized tonic-clonic; IQ intelligence quotient; S/T, spatial and temporal. 
temporal lobe, corresponding to the fornix and thalamic bundle (Thal-Bundle) (Fig. 5A, middle) and also posterior thalamic peduncle (Post-Thal-Ped) fibers (Fig. 5A, right). These and also the pyramidal pathway were isolated using appropriately placed regions of interest (ROIs), (Fig. 5, middle and right). A one-way analysis of variance (ANOVA) on the number of fibers from the hemisphere with the thalamic lesion (normalized to the contralateral one) (Fig. 5, Supporting Table 2) was performed to test the hypothesis of no significant difference in the patients' average fiber count between the four tracts. Paired comparisons between average fiber bundles were performed using t-tests.

\subsection{Steady-state visual evoked potentials (SSVEPs)}

To probe the cortical responses to external visual stimuli of the posterior cortex of the lesion hemisphere and to compare them with responses from the healthy hemisphere, we designed a protocol with sequential stimulation of the four quadrants of the visual field. SSVEPs similar to the ones of di Russo et al. [17] were used and consisted in the presentation of a set of four sinusoidal Gabor gratings with horizontal bar stimulus in a counterbalanced sequence in the four quadrants of the visual field (Fig. 6B). The patient was seated comfortably in a chair facing a fixation cross at the center of an LCD display with a refresh rate of $75 \mathrm{~Hz}$ while the Gabor gratings flickered in two blocks of 100 stimuli per quadrant with an interval between blocks of $15 \mathrm{~s}$ and in a counterbalanced sequence. Four stimulation frequencies were used $(5.4,9.4,12.5$, and $18.8 \mathrm{~Hz})$, corresponding to subharmonics of the fundamental LCD refresh cycle of $37.5 \mathrm{~Hz}$. For patients P3 and P4, only the 9.4- and $12.5-\mathrm{Hz}$ frequencies were tested because of limited collaboration.

After high-pass filtering of the EEG (electrode Oz with reference in $\mathrm{Fz}$ ) at $1 \mathrm{~Hz}$ and low-pass filtering at $70 \mathrm{~Hz}$, epochs corresponding to 10 cycles of the applied VEP stimulus were created and averaged for each quadrant of the visual field and frequency of stimulation. SSVEPs were then submitted to a FFT (fft function from Matlab R2013) to recover the first five harmonics. The signal-noise ratio (SNR) was calculated by dividing the peak amplitude by the average of the two neighboring spectral points [18].

\section{Results}

\subsection{Anatomical features of thalamic lesions}

An overall visual inspection of the thalamic lesions revealed that, in all cases, lesions were unilateral and predominantly near the ventricles, affecting mainly the medial and superior nuclei of the dorsal thalamus, with relative sparing of the more lateral ventral thalamus (Fig. 2). This topographic distribution of lesions produces a selective sparing of the first-order thalamic nuclei, which are localized more laterally and ventrally, with preferential involvement of higher-order nuclei [19].

The volumetric data for the thalamus are shown in Fig. 2 and reveal a wide range of volume loss from 19\% to a massive $94 \%$ (Supporting Fig. 1B). The comparison of volume loss to overall developmental status (Supporting Fig. 2B) demonstrated a trend for more severe delay in patients with large atrophy. Nevertheless, even a loss higher than $50 \%$ volume is compatible with a normal developmental status.

The pulvinar was also quantified and found to have a wide range of atrophy (Fig. 2), from 11\% to 74\% (Supporting Fig. 1B).

In the patients where an automatic comparison of cortical thickness between hemispheres was performed $(N=6)$, no asymmetry was found (Supporting Fig. 2A), which agrees with the lack of abnormalities in the visual MRI inspection. This is in contrast with the loss of white matter demonstrated by the enlarged ventricles on the hemisphere with the thalamic lesion in all these patients (Fig. 2).

\subsection{Electroclinical features}

For all patients, the CSWS was a focal one, with continuous spiking restricted to a region of the lesion hemisphere (Fig. 1). This remained stable in repeated whole-night EEG recordings of the same patient despite a strong trend to variable secondary propagation to other brain areas, including the contralateral hemisphere. This supports the interpretation that the epileptic spiking producing the CSWS pattern originates in a restricted cortical subregion.

The effect of the lesions on the physiological EEG rhythms revealed the following (Fig. 3): (a) decrease in power of the posterior alpha rhythm over the lesioned hemisphere, which in five out of nine patients is lower than the $50 \%$ interhemispheric ratio taken as the lower limit of normal in clinical practice; (b) for the Mu rhythm, this ratio remained above $50 \%$ in six patients and was absent bilaterally in two, and one patient (P3) had no demonstrable Mu rhythm over the lesion hemisphere only, which was associated with clinical evidence for upper motor lesion (discrete right lower limb spastic monoparesis); (c) the sleep spindles were consistently present in both hemispheres of all patients, with eight out of nine patients with pathological-healthy hemisphere AI within the limits reported [13] for partial epilepsy of $-23 \%$ to $+29 \%$. The spectra also showed a similar peak frequency for spindles in both hemispheres of each patient (Fig. 3B), supporting the interpretation that, in our series, this rhythm is largely preserved despite the large unilateral thalamic lesions.

\subsection{Sleep-stage correlations of epileptic activity}

The asymmetric pattern of continuous spiking allowed the manual scoring of sleep using the contralateral EEG channels in eight out of nine patients. For most patients, the overall structure of the sleep was not significantly disturbed, with an average of five cycles per night and sleep efficiencies in the normal range (>90\%) for all patients. This supports the interpretation that the continuous spikes overlap, but do not significantly disturb, the normal succession of sleep stages and is in line with the absence of excessive daily sleep complains in all the patients.

The quantification of the SI for each sleep stage for one night revealed a persistent increase of this index in all non-REM stages (1, 2 , and 3) compared with wakefulness and REM sleep (Supporting Fig. 2C), which is the paramount characteristic of the CSWS.

\subsection{Source analysis}

The average reference maps for representative spikes produced dipolar fields with well-defined peaks over the posterior cortex for the nine patients, stable between half-peak and peak and always lateralized to the lesion hemisphere (Fig. 4, left maps). In the eight patients where it was computed, the Laplacian produced sharper configurations of sources and sinks that were largely compatible with the average maps but improved their spatial resolution (Fig. 4, middle maps). The SLORETA, with the anatomical space of solutions restricted to the cortex segmented from the MRI, produced maximum scores in well-defined areas (Fig. 4, right maps) that are largely compatible with the spatial configuration of the previous scalp maps but which additionally support a deep localization for most patients. The solutions for the nine patients effectively favor the origin of spikes in a posterior brain cortical patch, which show reduced spatial variation from half-peak to peak.

\subsection{Tractography}

The fiber quantification of the fornix, Thal-Bundle, Post-Thal-Ped, and pyramidal pathways puts in evidence an interhemispheric asymmetry, with loss of fibers in the first two pathways and a lesser one in the last two (Fig. 5B). The one-way ANOVA for the normalized fiber count (Supporting Table 2) produced an $F=4.743$, leading to the 


\section{P1(8y)}

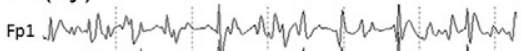

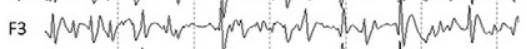

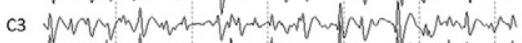

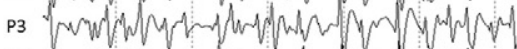

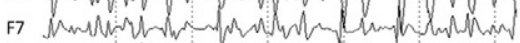

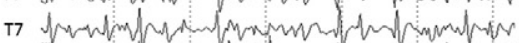

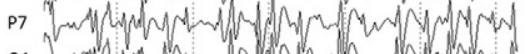

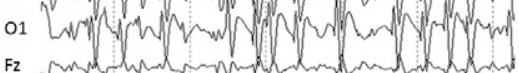

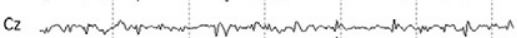

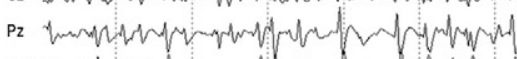

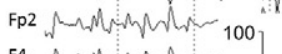

F4 Mumpupura

C4 surmanpmon

P4 numwarmoun 50

F8 wumpropher

T8 Anmpinporis

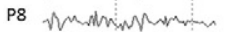

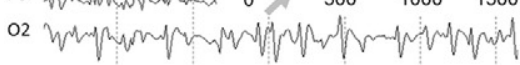

P4 (9y)

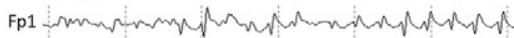

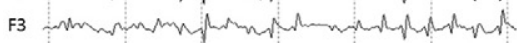

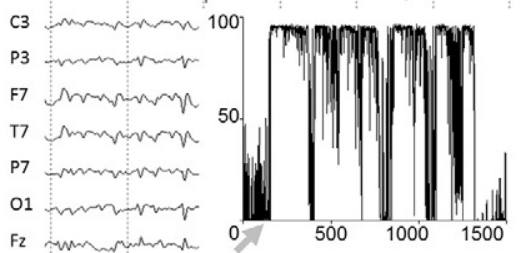

$\mathrm{Cz}$

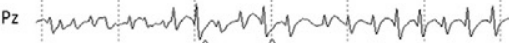

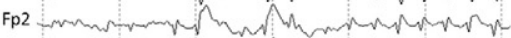

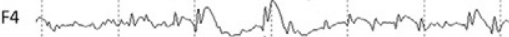

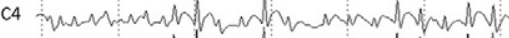

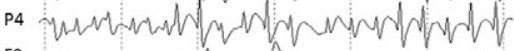

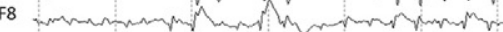

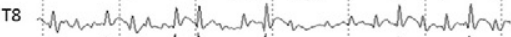

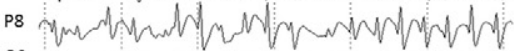

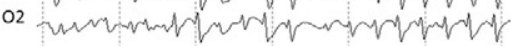

P7 (2y)

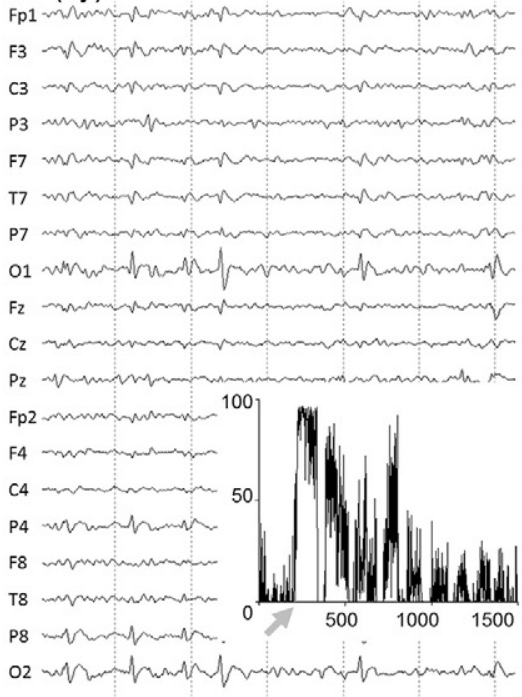

P2 (11y)

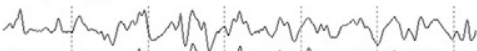

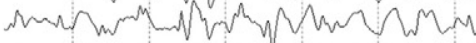

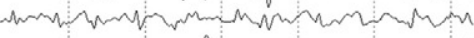

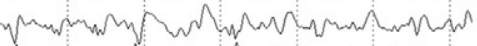

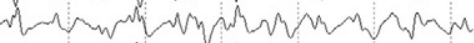

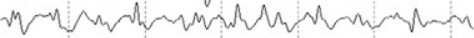
Whanding

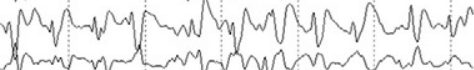

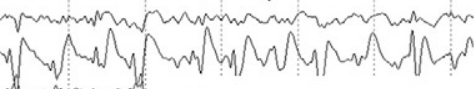

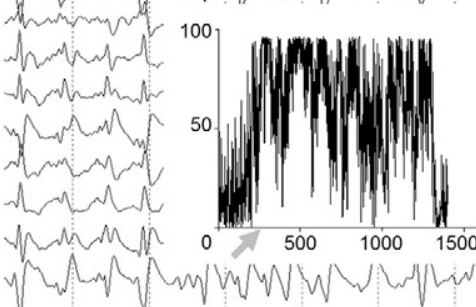
P5 (8y)

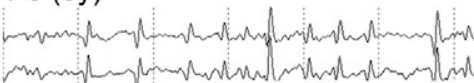
(100)
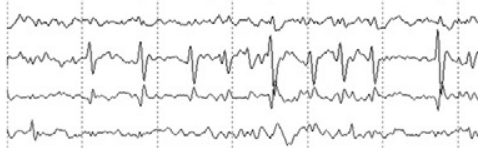

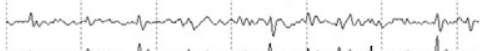

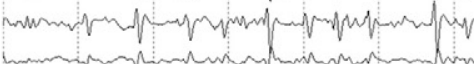

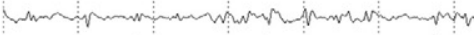

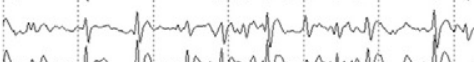

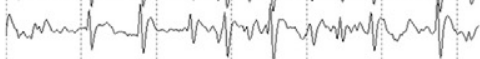

P8 (9y)

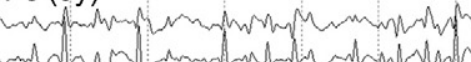

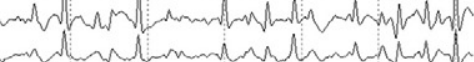

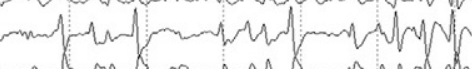

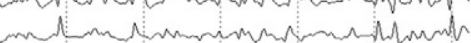

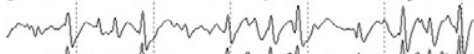

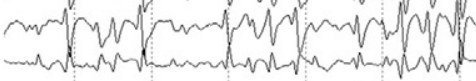

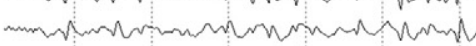

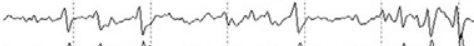

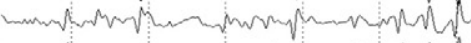

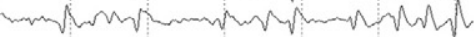

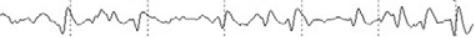

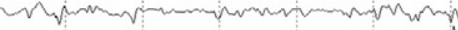

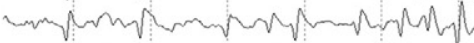

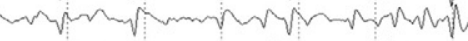

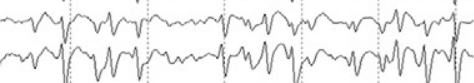

P3 (4y)

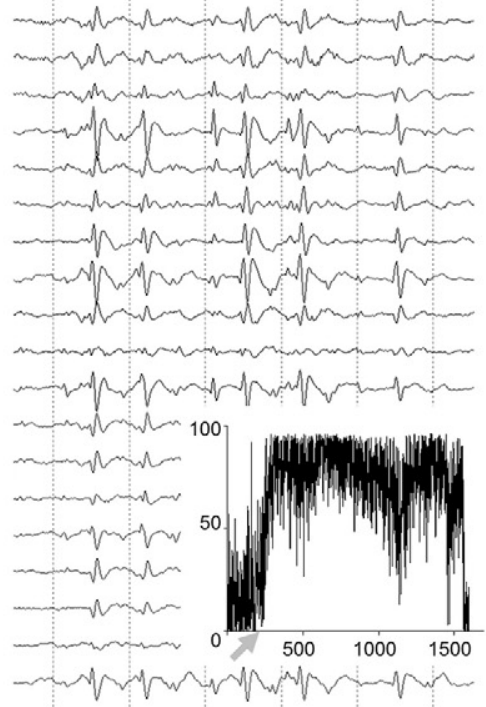

P6 (8y)

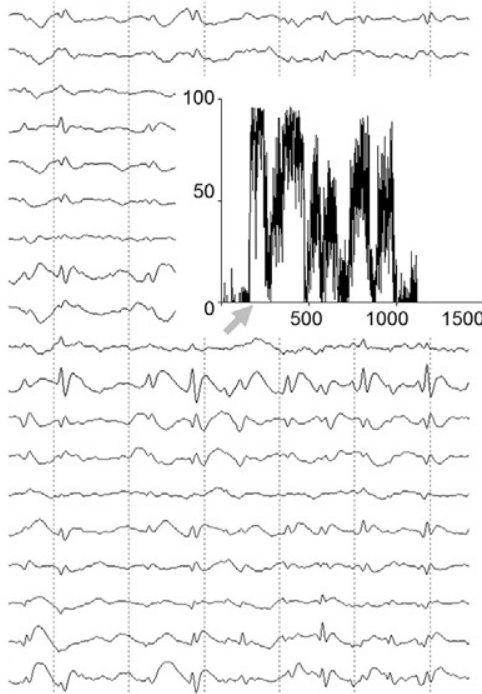

P9 (9y)

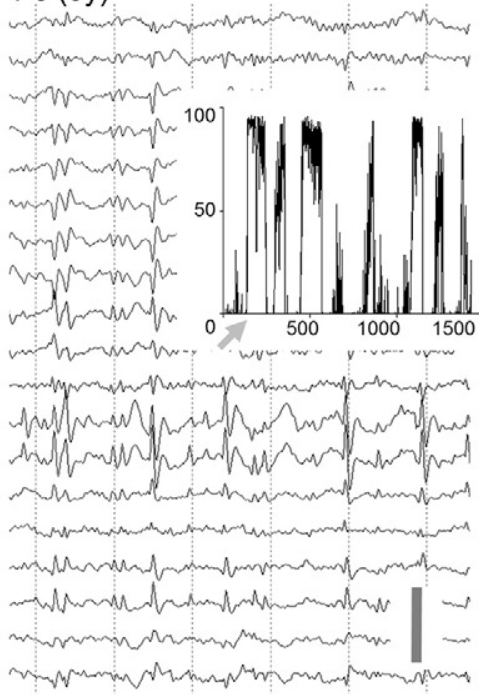

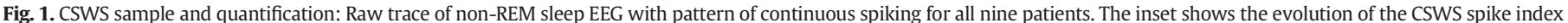
through a whole night. Dotted vertical lines correspond to 1-s period, whereas the amplitude vertical bar is $150 \mu \mathrm{V}$. 

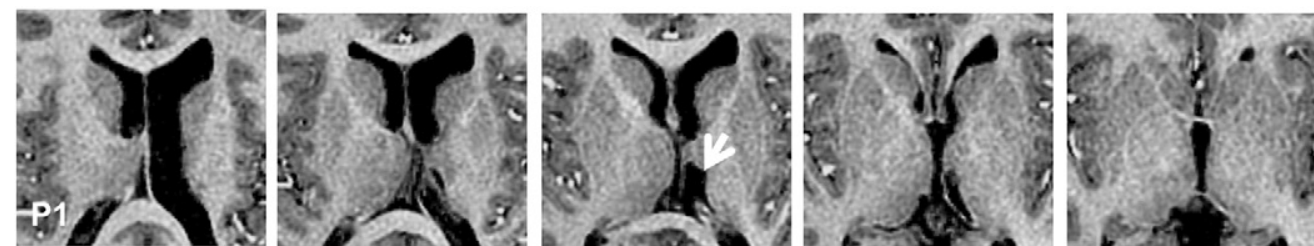

Thlamus (cc)

Left-2.80(44\%)

Right-6.29
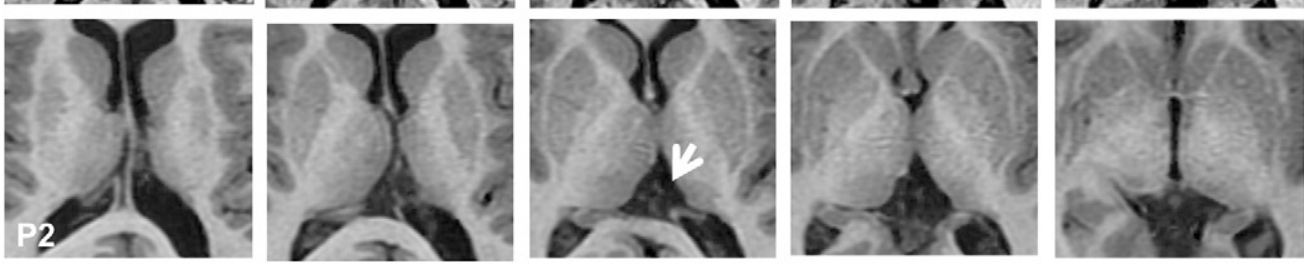

Pulvinar (cc):

Left-1.01(61\%)

Right-1.65
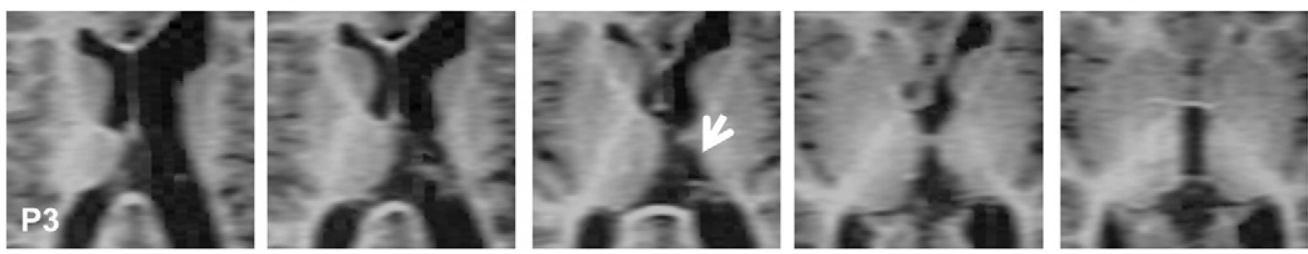

Thlamus (cc):

Left-4.02(71\%)

Right-5.65

Pulvinar (cc):

Left-1.08(72\%)

Right-1.51
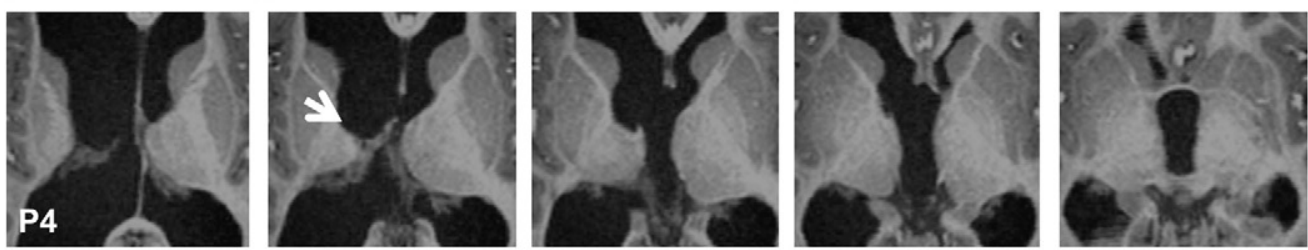

Thlamus (cc):

Left-1.74(31\%)

Right-5.60

Pulvinar (cc):

Left-0.24(16\%)

Right-1.54
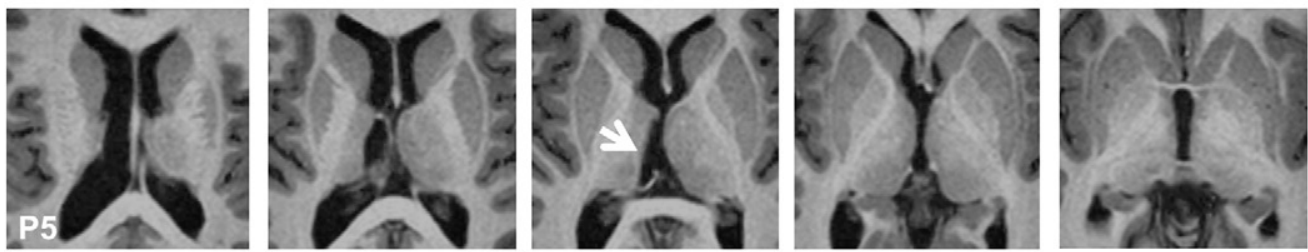

Thlamus (cc):

Left-7.45

Right-4.09(55\%)

Pulvinar (cc):

Left-1.92

Right-0.92(48\%)
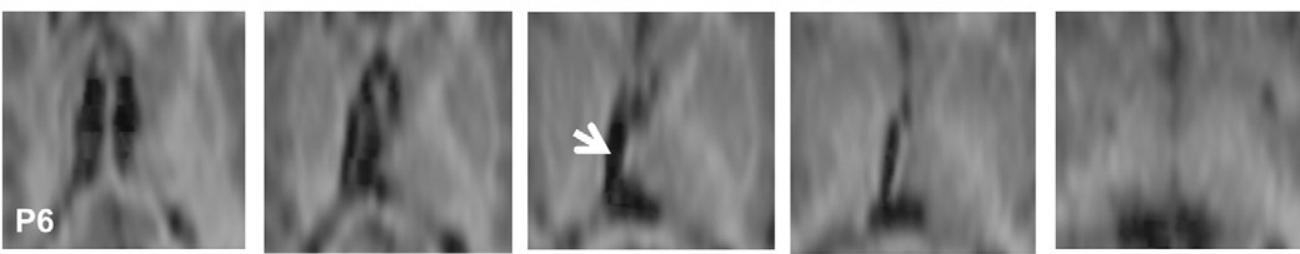

Thlamus (cc)

Left-7.51

Right-4.43(59\%)

Pulvinar (cc):

Left-1.46

Right-1.30(89\%)
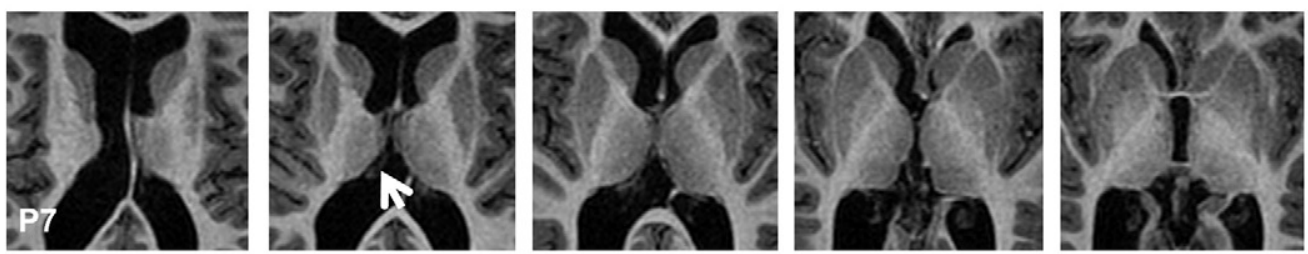

Thlamus (cc):

Left-4.07

Right-0.18(6\%)

Pulvinar (cc):

Left-0.47

Right-0.33(70\%)
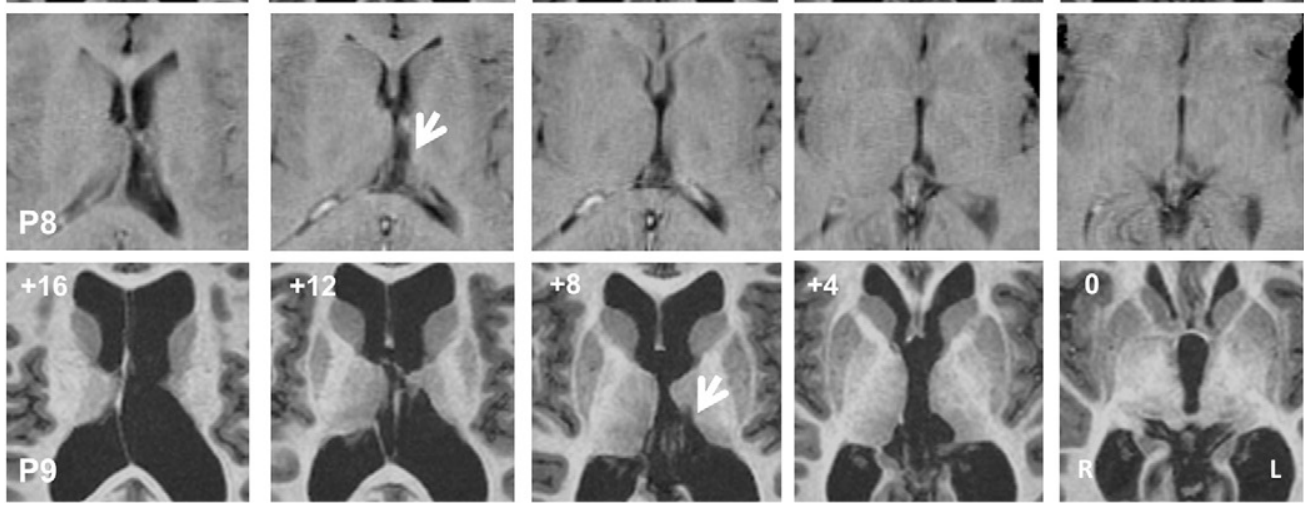

Thlamus (cc):

Left-4.81

Right-4.19(87\%)

Pulvinar (cc):

Left-1.57

Right-1.32(84\%)

Thlamus (cc):

Left-2.28(47\%)

Right-4.84

\section{Pulvinar (cc):}

Left-0.49(88\%)

Right-0.56

Thlamus (cc):

Left-2.85(58\%)

Right-4.93

Right-1.48

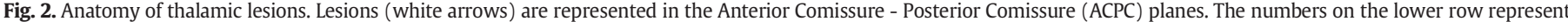

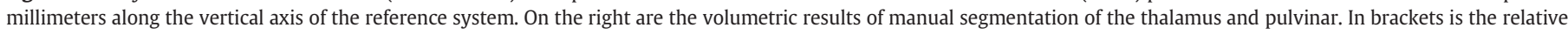
volume of the structure with the lesion compared with the unaffected one in the healthy contralateral hemisphere. 
A)

Alpha rhythms

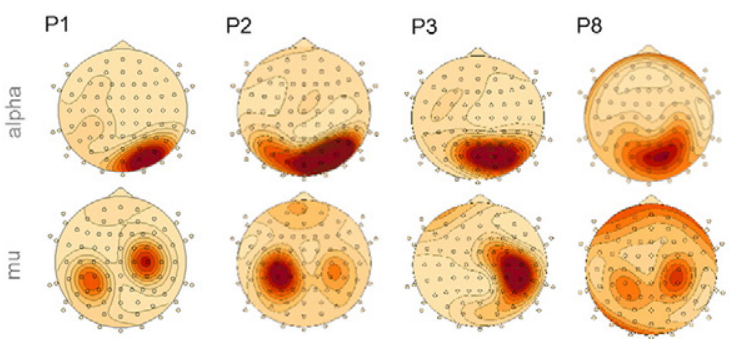

left thalamus lesion
P9

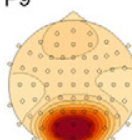

P4

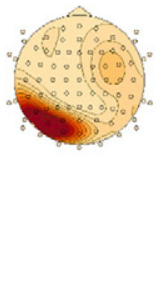

P5

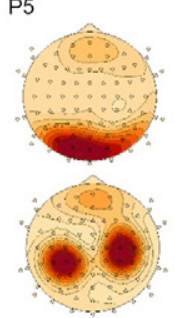

P6

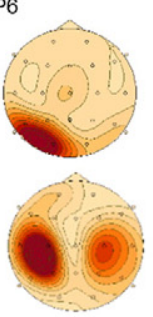

P7

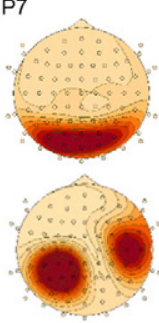

right thalamus lesion

B)

$\bar{\Sigma}$

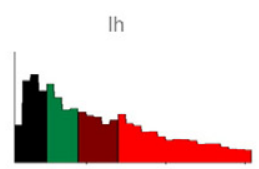

ล

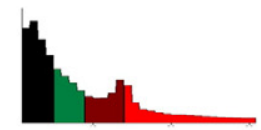

@

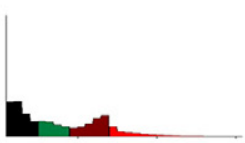

$\stackrel{\infty}{\infty}$
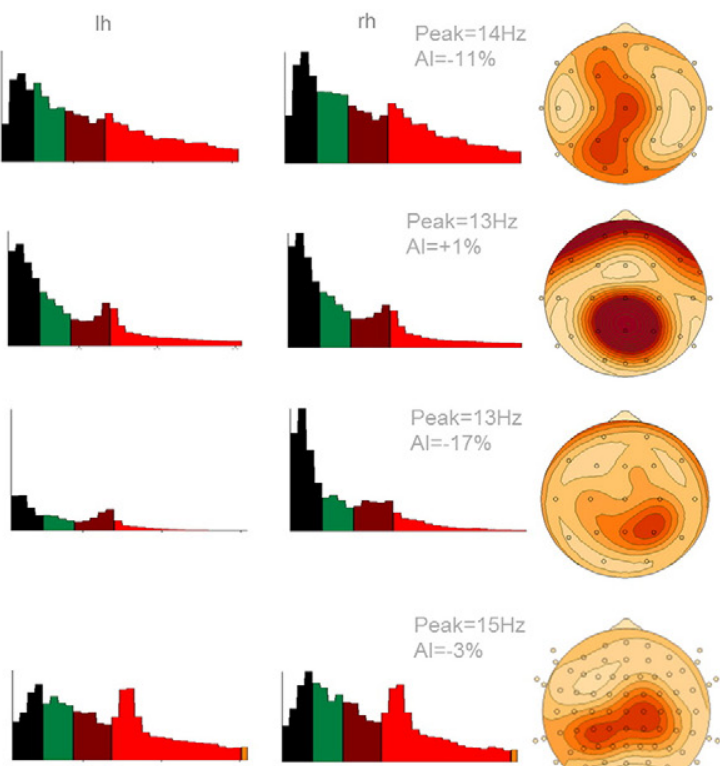

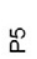
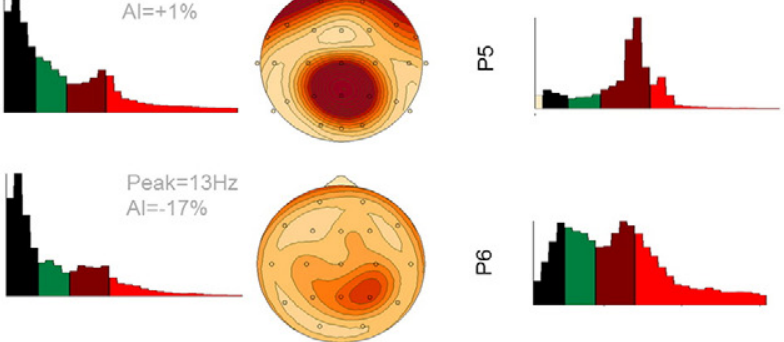

$\therefore$
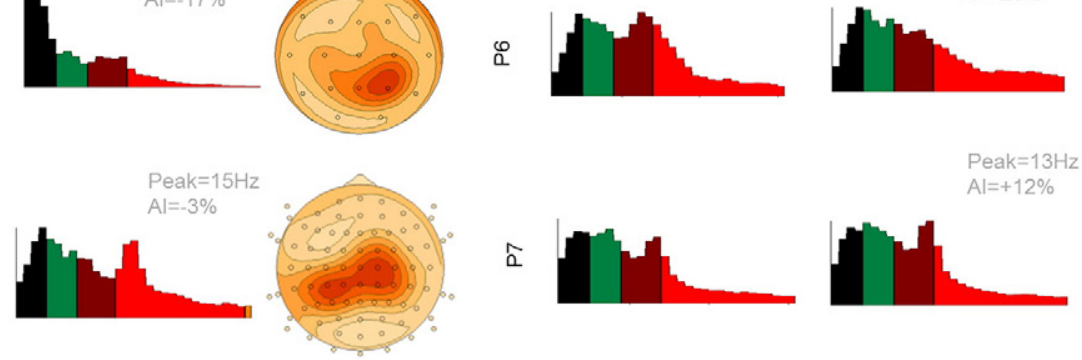
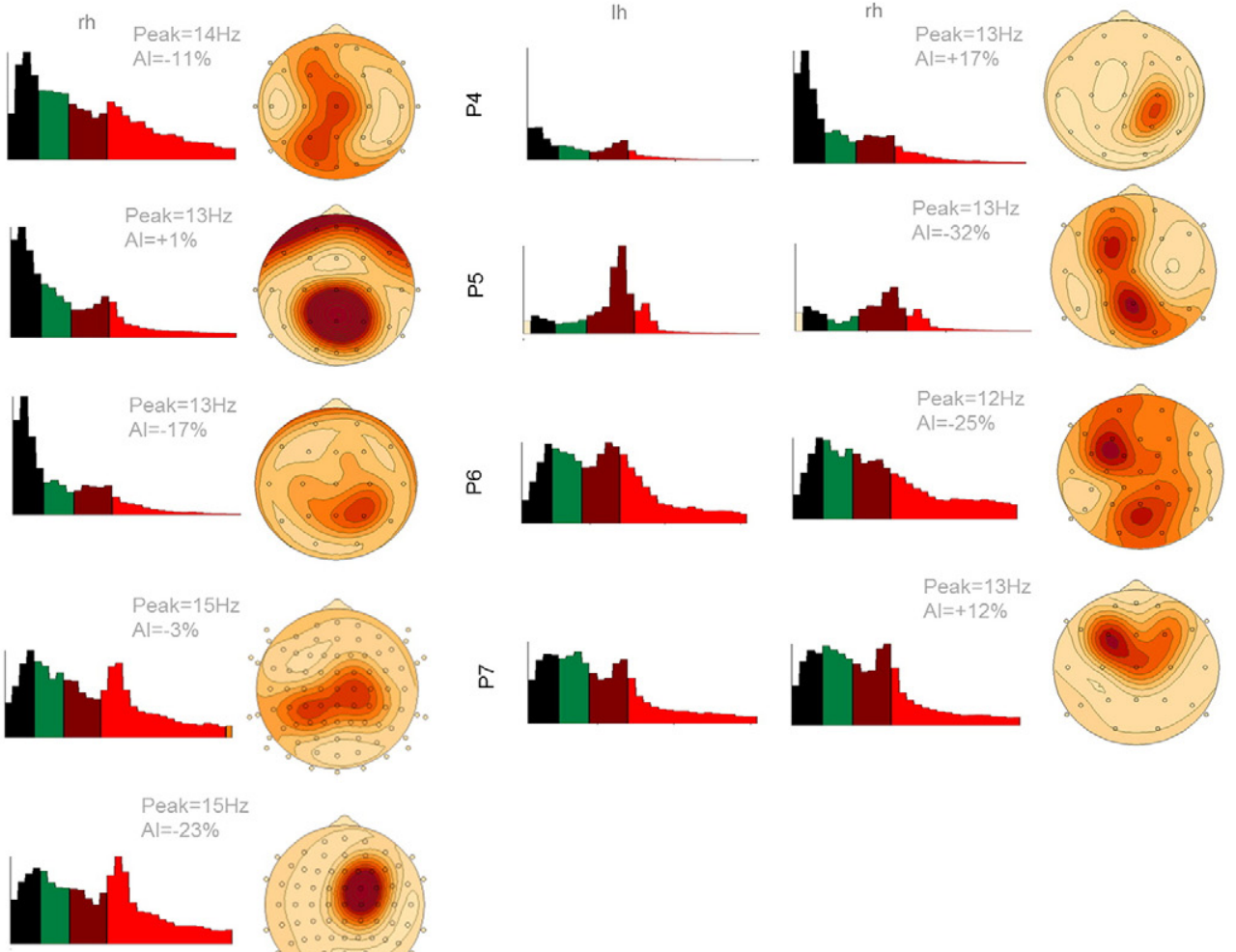

\&
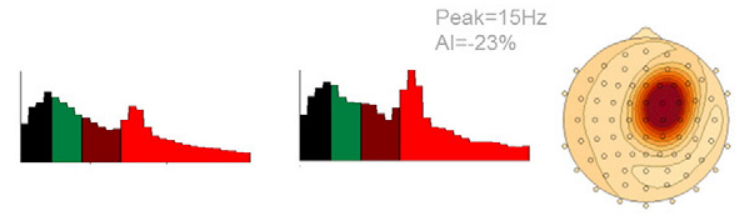

C)
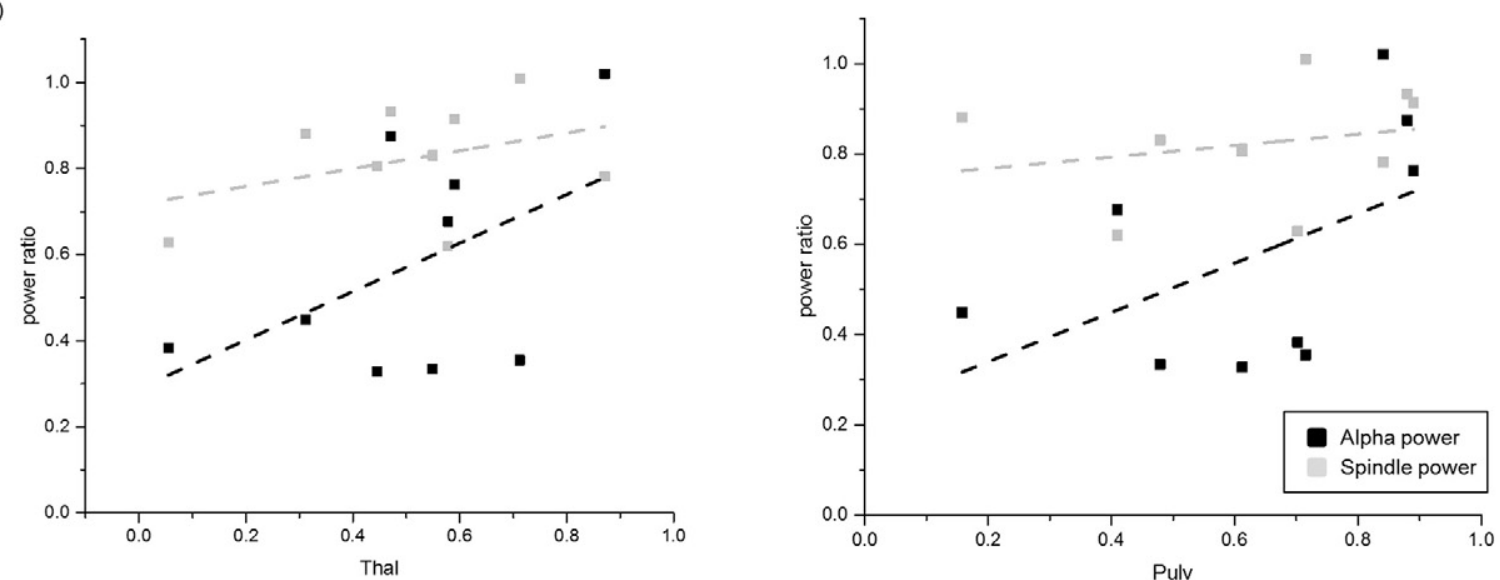

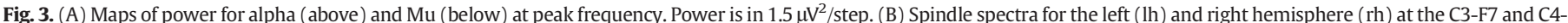

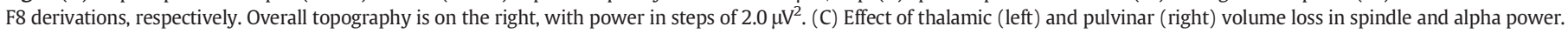
Changes in thalamic and pulvinar volume affect the alpha but not the spindle rhythms. Power is normalized to the healthy hemisphere. 


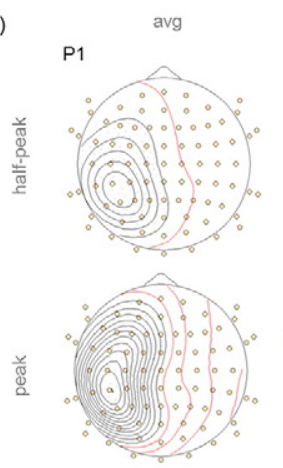

P3
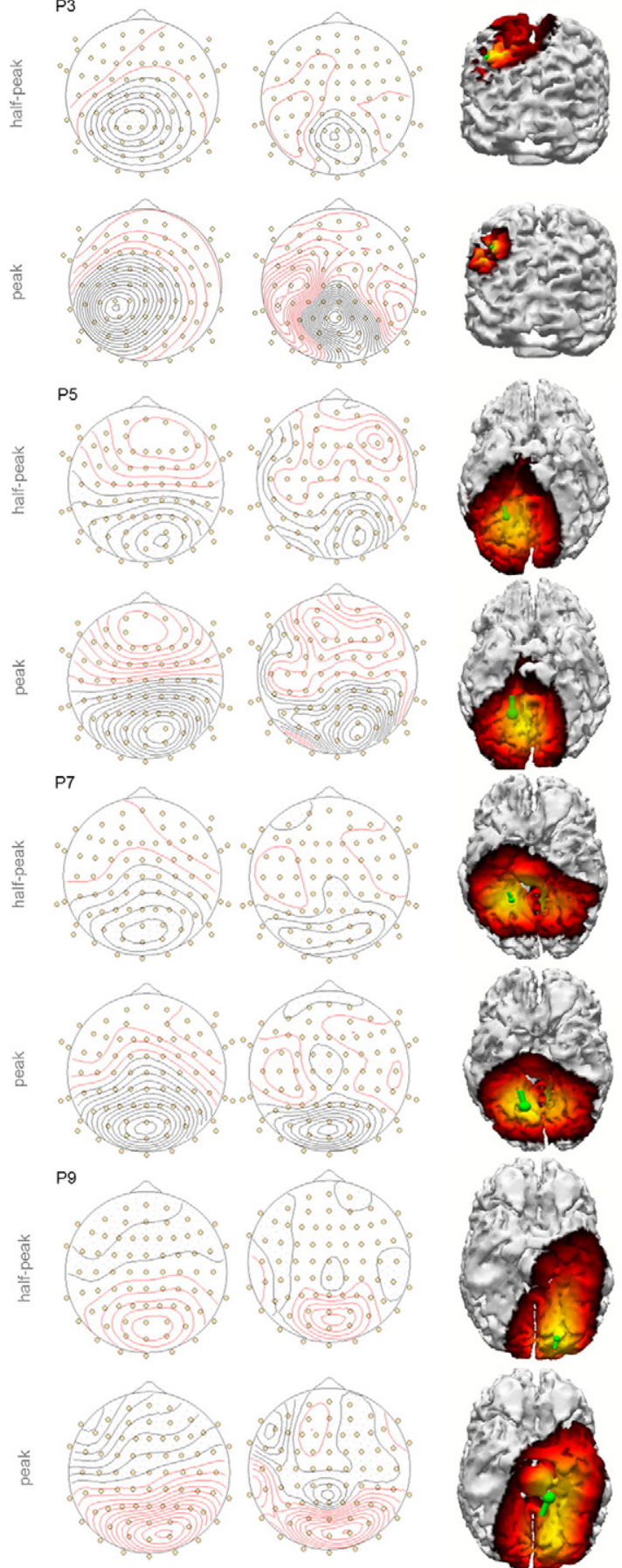
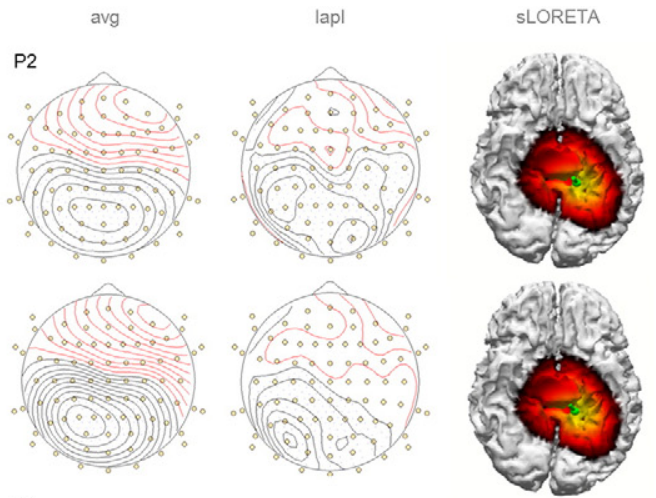

P4
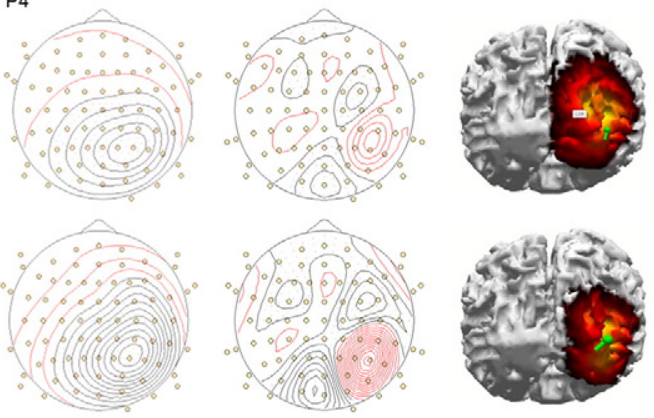

P6
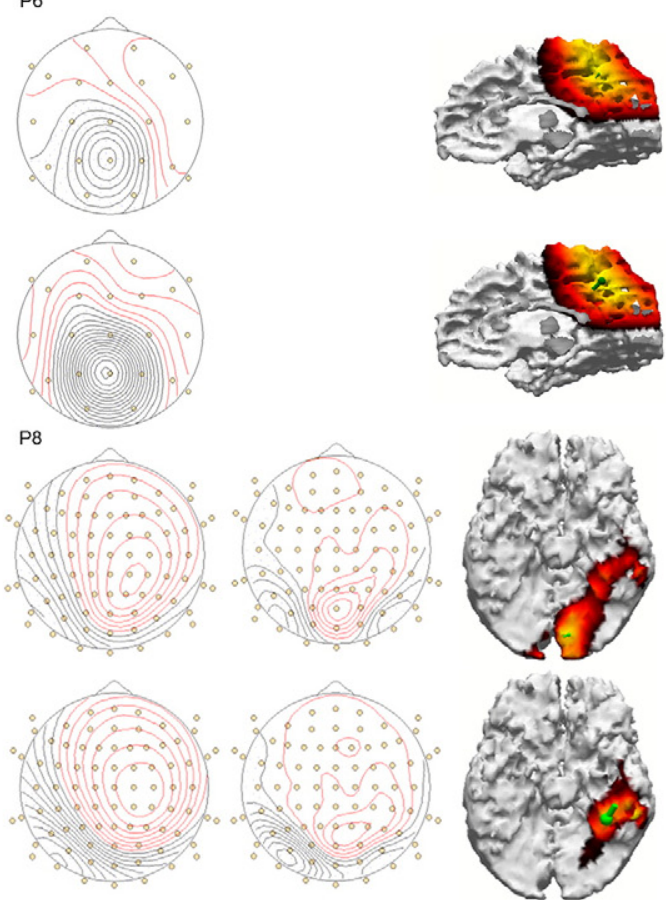

B)
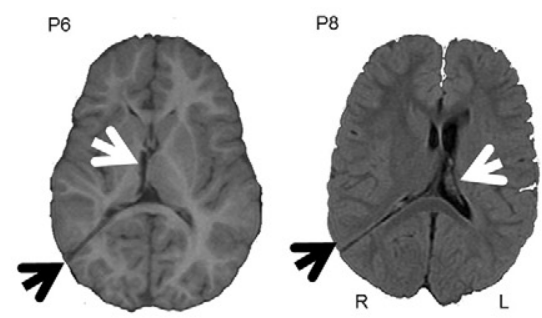

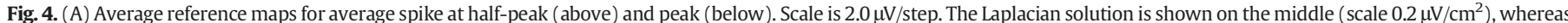
the sLORETA sources are on the right. (B) Brain MRI of patients with VPSs (black arrows) and relationship with thalamic lesion (white arrows). 
refusal of the null hypothesis of no difference in mean ratio between the four bundles with a $P<.02$. Paired comparisons of the means of the four bundle types using paired $t$-tests revealed a significant difference between the pyramidal and both the fornix $(P<.02)$ and Thal-Bundle $(P<.02)$ but not between the last two bundles $(P<.36)$. The Post-
Thal-Ped demonstrated a clear fiber loss in the hemisphere with thalamic lesion in only two patients, and the $t$-test in the comparison with the pyramidal pathway failed to reach significance $(P<.11)$. Overall, these results demonstrate that the loss of white matter associated with the thalamic lesions produces loss of fibers which is significantly

A)

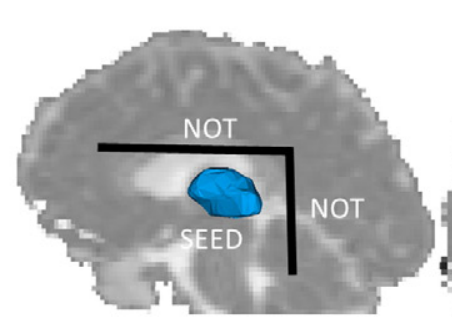

B)
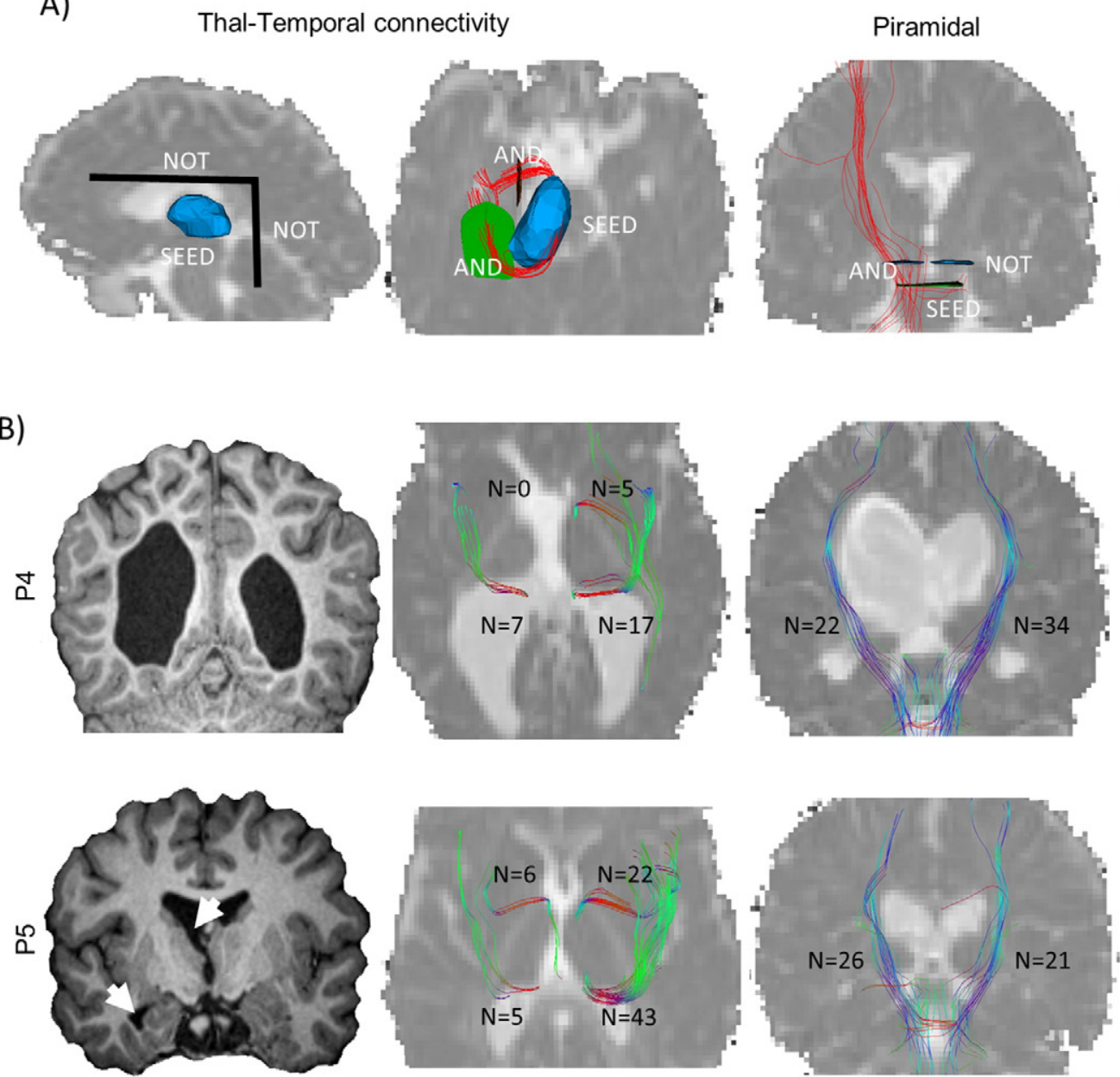

Piramidal

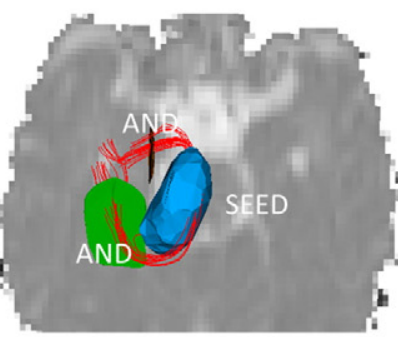

\section{Thal-Posterior connectivity}
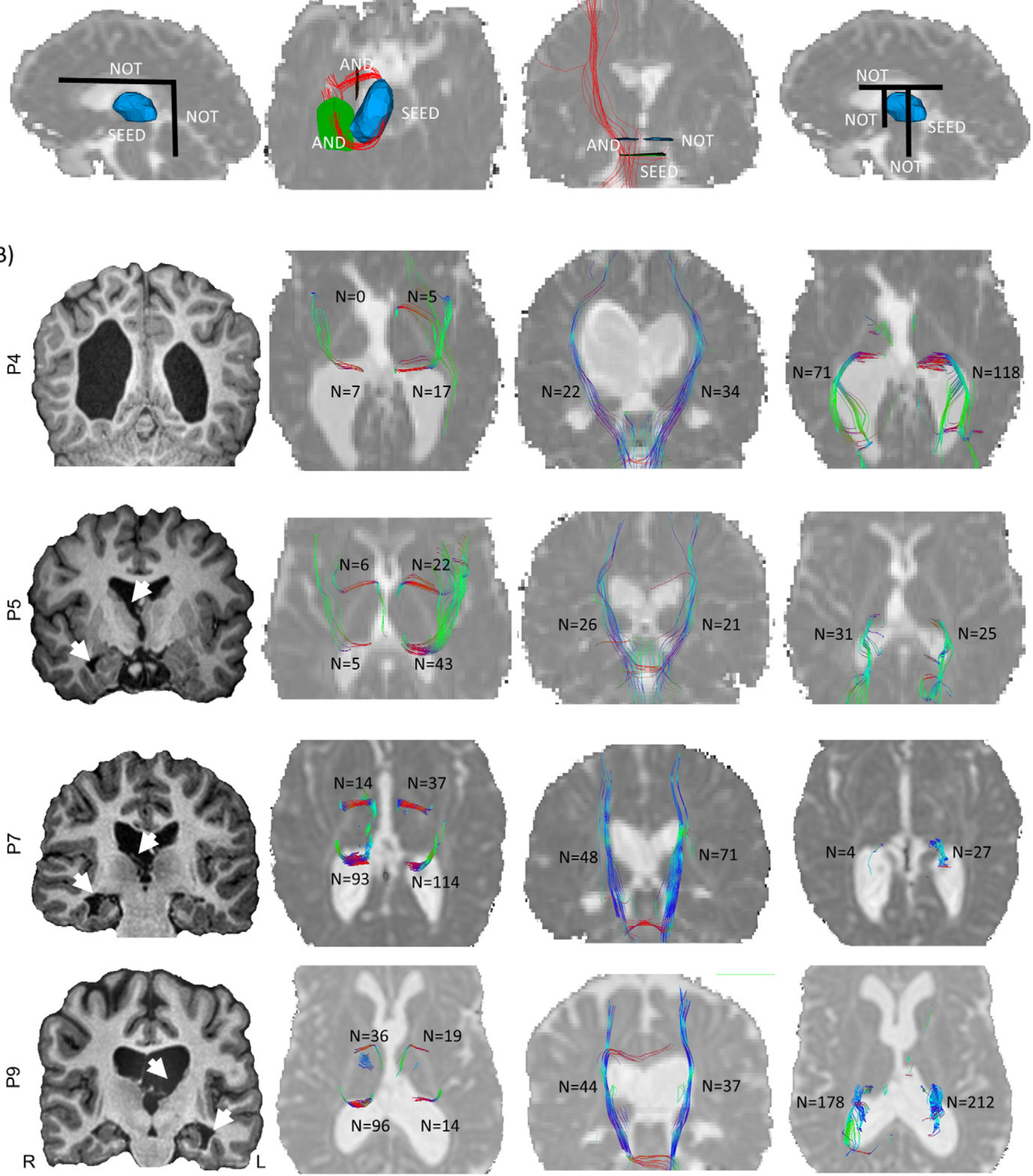

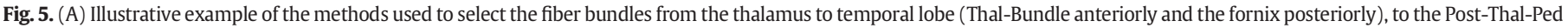

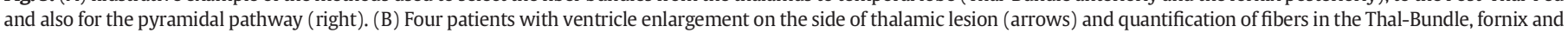
Post-Thal-Ped. The pyramidal pathway was also quantified as nonthalamic control. 

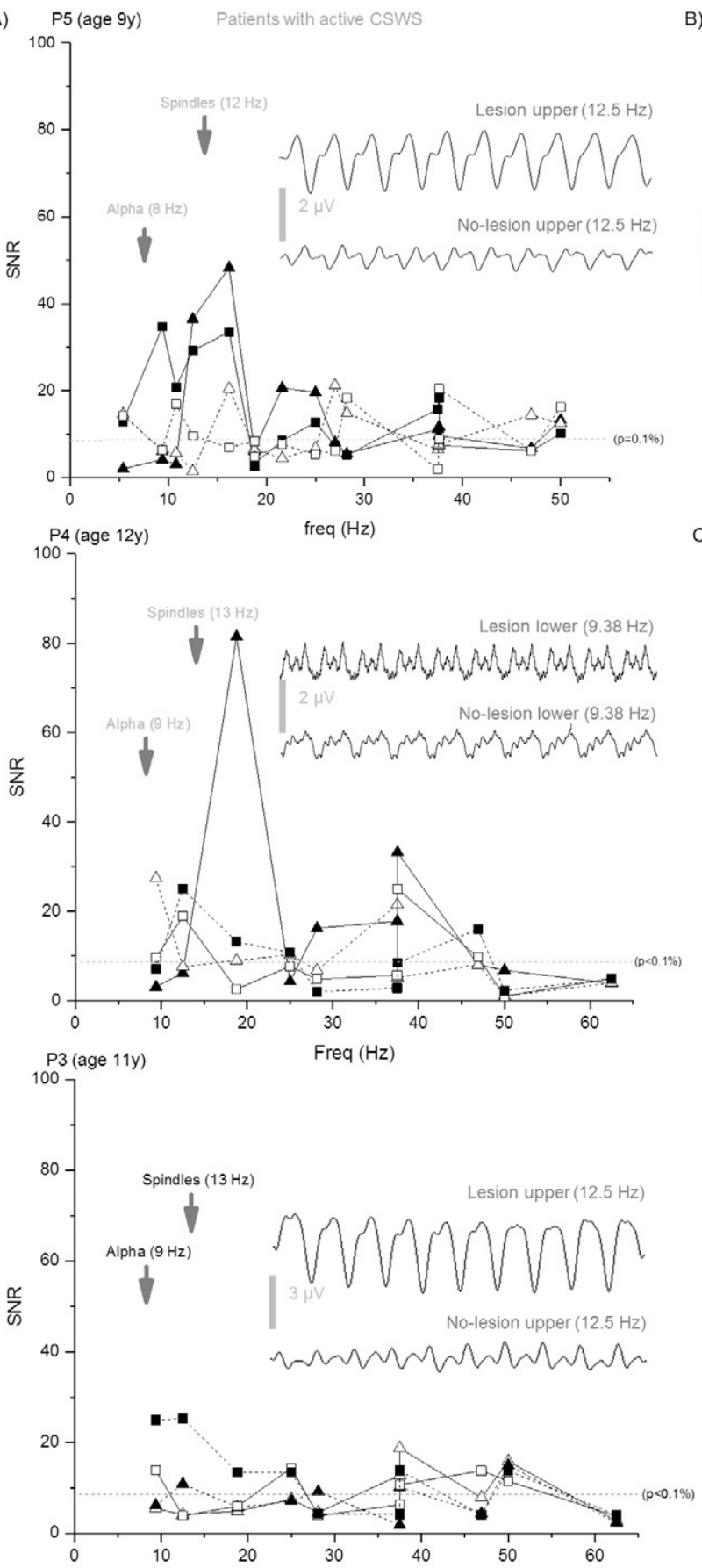

D)

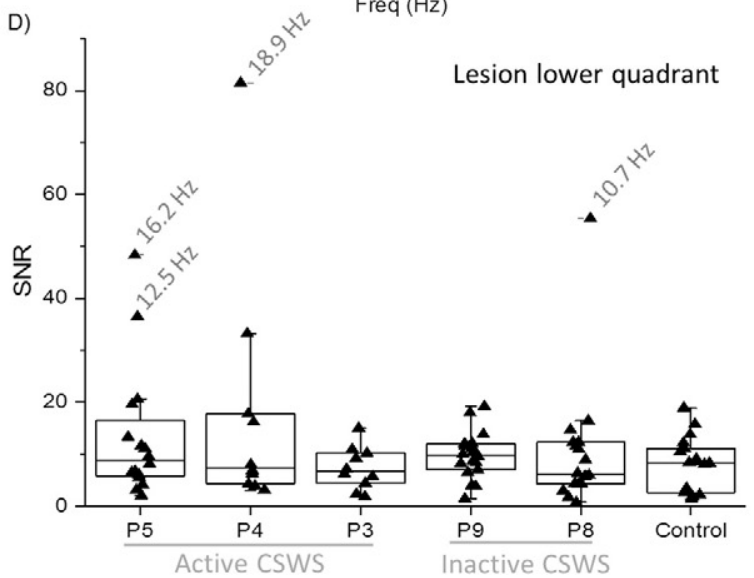

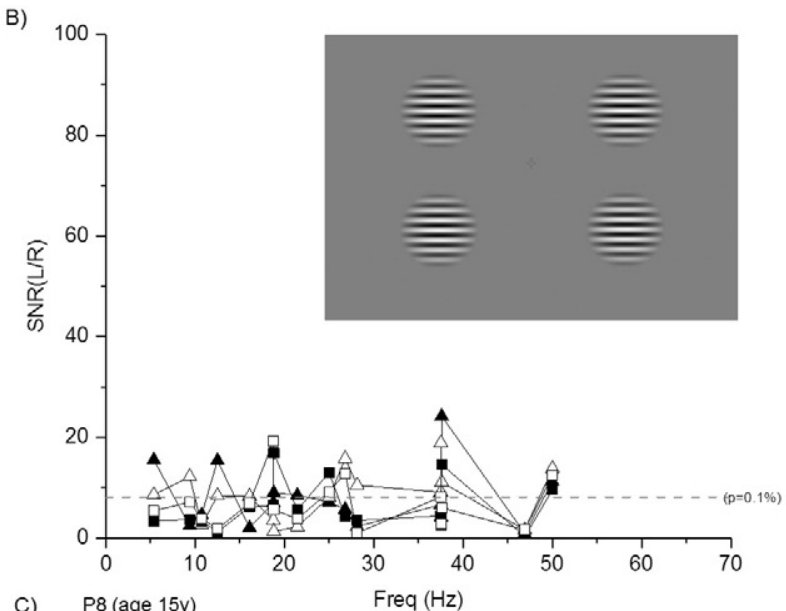

C)
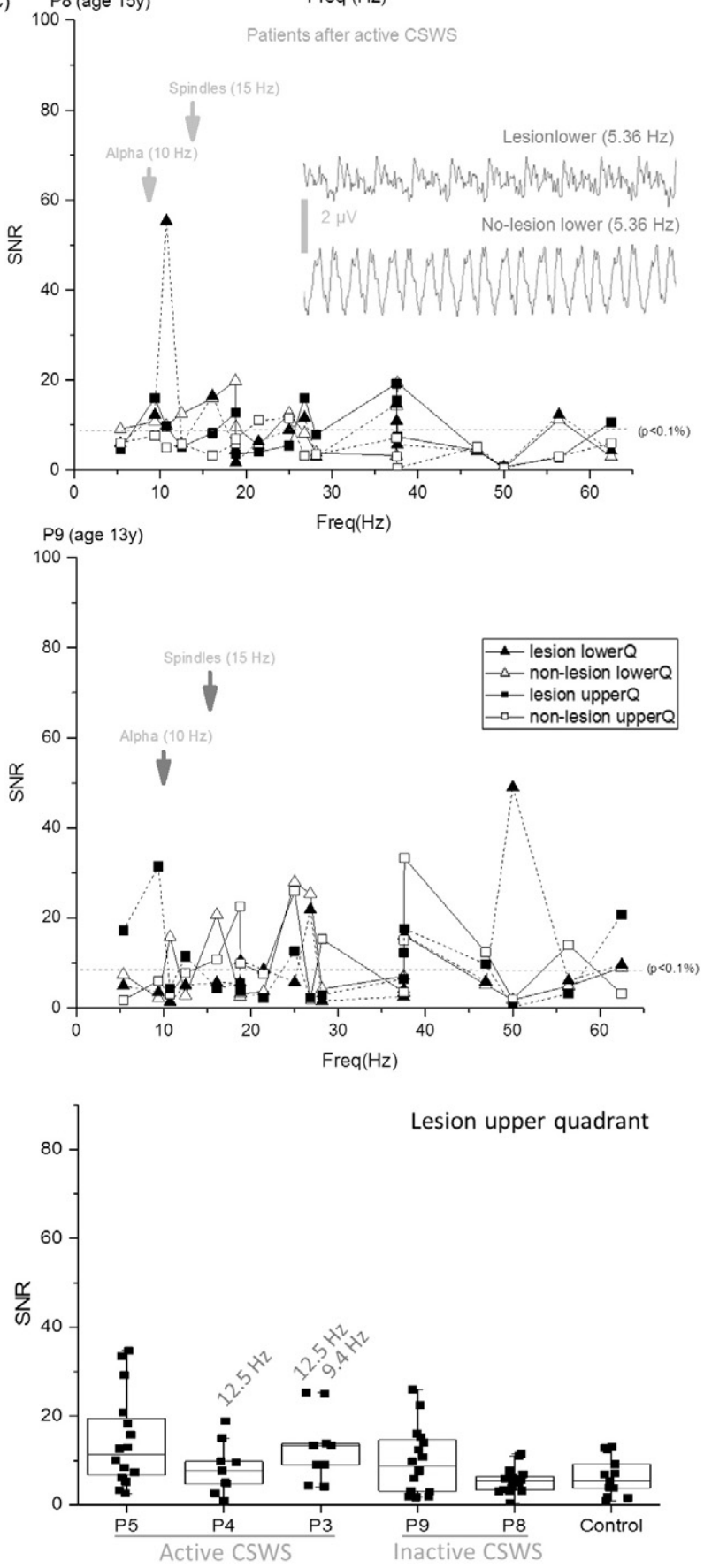
more important for thalamic-cortical projections than for nonthalamic ones (pyramidal pathway).

\subsection{SSVEPS}

The three patients tested with active CSWS (Fig. 6A) presented enhanced responses to stimulation of the lesion hemispheres on the 9- to $19-\mathrm{Hz}$ frequency range, which is reflected in the positive outlier values of Fig. 6D, whereas no such asymmetry was apparent in other frequencies of stimulation or in the healthy subject (Fig. 6B). Even though no epileptic spikes were present throughout the SSVEP protocol, these observations support a frequency-dependent increased excitability of the cortex in these patients. The increased excitatory responses are present in both quadrants in patient P4 and are restricted to the lower quadrant in patient P5 and to the upper quadrant in P3.

Two patients were tested after resolution of the CSWS pattern (Fig. 6C), and except for one (P8) where an asymmetry in SSVEPs could be demonstrated around the peak frequency of the alpha rhythm (Fig. 6D), no abnormal increases in excitatory responses were apparent. This observation supports a partial recovery of the SSVEPs responses towards normality and no evidence for an increased excitability at the spindle frequency range in the hemisphere with the thalamic lesion.

\section{Discussion}

\subsection{Comparison of clinical features with data from literature}

The clinical features in our series of patients with CSWS and thalamic lesions are similar to the ones described by early authors [6-8] and demonstrate the existence of an age- and sleep-stage-dependent dysfunction of the thalamocortical circuitry similar to the one observed in other etiologies of CSWS [20]. The analysis of individual MRI features of lesions in those case reports puts in evidence the predominant involvement of medial and dorsal thalamic nuclei in line with the one in our series with which they share a common etiology.

Comparison of our results with larger series $[5,9,10]$ is more difficult because the multiplicity of etiologies and the large extrathalamic lesions in most of their patients precluded the demonstration of a clear anatomical-physiological model. Their predominant lesions involved the lateral and ventral thalamus, in association with much larger vascular lesions of the middle cerebral artery, which is quite distinct from the medial and dorsal localization of exclusively thalamic lesions in our series.

A trend for larger thalamic lesions to have an impact on development is apparent in our series (Supporting Fig. 2B) and can also be found in previous case reports of patients with larger thalamic destruction (case 1 of Incorpora et al. [6] and the case of Monteiro et al. [7]) compared with the more favorable outcome of patients with more limited lesions.

\subsection{Comparison of thalamic lesions with other similar series}

The thalamic hemorrhage underlying the lesions in our series is similar to the one described previously $[21,22]$, mostly with unilateral incidence. Seven of our nine patients had clear history of unilateral thalamic hemorrhage, whereas the two patients with no clear etiology (P1 and P5) had very similar lesion types, suggesting a common cause, possibly occurring before birth. The presence of hemosiderin in the MRI of patient P5 supports this suggestion (Supporting Fig. 1).

Regarding the predominant distribution of lesions in the thalamus, Kersbergen et al. [11] described mostly periventricular localizations (8/14 patients), as was the case in the early case reports $[6,7,8]$, in line with a similar distribution in all our cases (Fig. 2). This suggests that the medial dorsal thalamus in contact with the ventricles is most commonly affected, with a relative sparing of more lateral and ventral thalamic regions. In Losito et al. [10], when the thalamic lesion involved predominantly the medial-dorsal nuclei, there was a higher incidence of CSWS (61\%) than when this was not the case (39\%), suggesting that this particular distribution of lesions is more prone to produce the CSWS, in line with our observations.

The overall pattern of lesions in our patients suggests a predominant involvement of higher-order thalamic nuclei [19], leading to a selective deafferentation of cortical association areas from their thalamic connections, in comparison with first-order nuclei connecting to primary somatic-sensory cortex. This observation correlates well with the reported preservation of both the Mu rhythms and spindles on the affected hemisphere and also with the predominant incidence of the CSWS over association cortex reported using positron emission tomography in seven patients with the syndrome [23]. The preservation of sleep spindles along the so-called core pathway [24] is also in line with the functional integrity of sensory and visual thalamocortical relays and the absence of afferent somatic-sensory and visual deficits in our patients.

\subsection{Correlation of cortical origin of spikes and thalami lesions}

In all the patients of our series, a single cortical area in the lesion hemisphere was associated with the generation of the continuous spiking typical of the CSWS syndrome. In the two patients with VPSs, the spike source was distant from the cortical scar (Fig. 4B), suggesting that this does not play a role in the genesis of the continuous spike pattern of CSWS.

The widespread enlargement of ventricles on the side with thalamic lesion, together with the associated reduction of white matter and selective loss of thalamocortical fibers, supports an important cortical disconnection of the hemisphere with the sleep-related continuous spiking. During wakefulness, this disconnection does not lead to a clear increase in excitability, as spikes are absent or very scarce and the overlying physiological alpha rhythms are predominantly depressed in power (Fig. 3A). This observation is in line with data from preparations with cortical slabs disconnected from the thalamus in vivo [24], where a similar absence of spontaneous epileptic activity was demonstrated. The depressed alpha rhythm is similar to the one described after thalamic infarction [25] and compatible with animal deep electrode recordings where partial coherence analysis of this rhythm revealed a strong contribution from the pulvinar nucleus [26].

Despite this reduced spontaneous activity, the SSVEP stimulation revealed a frequency-dependent pathological increase in excitability to visual stimuli presented to the lesion hemisphere for all the patients tested (Fig. 6A, C, and D), as compared with the responses to the unaffected one, between 10 and $20 \mathrm{~Hz}$. Such frequency-dependent increase in excitability is not a general phenomenon in neocortical epilepsies as demonstrated by the distinct results of a corticocortical stimulation study in patients with cortical dysplasia [27] where the responses peaked for stimuli around $50 \mathrm{~Hz}$ and inhibition was apparent at

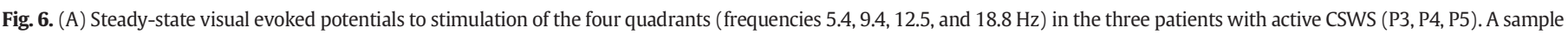

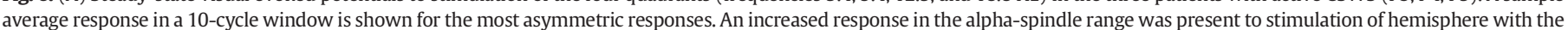

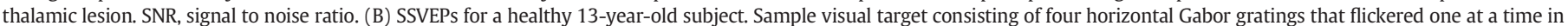

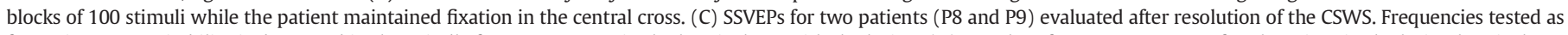

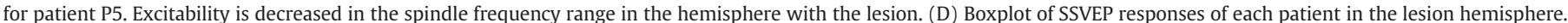

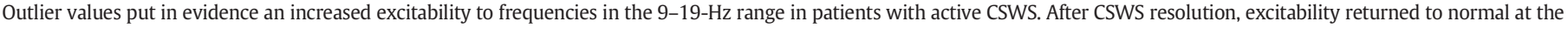

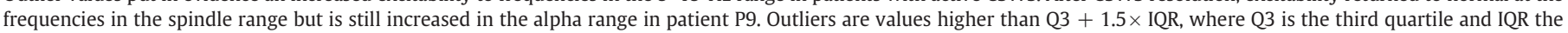
interquartile range. 
frequencies of $12.5 \mathrm{~Hz}$. It is also not present in our healthy volunteer or in the more extensive normative study of di Russo et al. [17]. The increased cortical excitability in the lesion hemisphere is restricted in spatial extent, as demonstrated by the involvement of a single quadrant of the visual field stimulation of the hemisphere with the lesion, either in patients with active CSWS or in patients with no active CSWS (Fig. 6D). In this hemisphere, we can still recognize the physiological spindles with preserved symmetry to the contralateral hemisphere (Fig. 3B). Because the frequency range of the peak SSVEPS reactivity overlaps the one of physiological spindles, the latter can potentiate this abnormal form of plasticity in the non-REM sleep in the patients with active CSWS (Fig. 6A). The SSVEPs of the two patients studied after resolution of CSWS no longer demonstrate such asymmetrical reactivity to afferent stimulation at the spindle frequency (Fig. 6C and D), supporting the interpretation that the sleep-induced excitability to spindles is no longer present.

\subsection{Influence of thalamic lesions in cortical plasticity}

The augmenting response was described [28] as increased cortical responses associated with repetitive sensory afferent stimulations with an optimal frequency around $10 \mathrm{~Hz}$ [29]. Other experiments demonstrated that augmenting responses to corticocortical stimulation were difficult to obtain in the neocortex of intact animals but were much facilitated after thalamic destruction [30]. The augmenting response is also dependent on the neocortical cholinergic tone, attenuating when this is high [31], which is the typical case during awakening and REM sleep. Extrapolating from these experiments to the unilateral brain corticothalamic disconnection of our patients, we postulate that thalamic deafferented neocortex should express an increased susceptibility to this type of frequency-dependent plasticity with corticocortical interactions when compared with the intact contralateral cortex.

The combination of the previous observations suggests that a pathological augmenting response type of cortical plasticity could provide an explanation for the peculiar and frequency-dependent increased excitability of the neocortical areas disconnected from the thalamus in our patients, and it could also explain the variation throughout the wakesleep cycle (Fig. 1), with peaks in conditions with low neocortical cholinergic tone, such as the non-REM sleep stages [32], and disappearance in stages where this tone is high (wakefulness and REM sleep). The demonstration, using the SSVEPs protocol in the three patients with active CSWS, of a frequency-dependent increased excitability to corticocortical stimulation in the $10-$ to $20-\mathrm{Hz}$ range over the lesion hemisphere (Fig. 6A) supports this proposal.

Sleep spindles are a physiological type of augmenting response, which is preserved during sleep in the lesion hemispheres of our patients (Fig. 3B), and can provide a cortical stimulus at the appropriate frequency to potentiate the pathological cortical augmenting response [33], eventually leading to the continuous spiking of CSWS [30]. Supporting this hypothesis is the observation that, in the few cases of CSWS submitted to functional hemispherectomy, a surgical procedure that disconnects the cortex from thalamic inputs and therefore of spindles, a rapid disappearance of the continuous spiking was observed [34, 35], suggesting that the cortical epileptic activity is dependent on the connection to the thalamus. The disappearance of the frequencydependent excitability at the spindle frequency in our two patients tested after resolution of the CSWS (Fig. 6C) is compatible with the previous hypothesis.

\subsection{A model for the origin of CSWS}

The overall electroclinical and anatomical features of patients in our series overlap the ones reported in similar cases from the literature, suggesting that a common mechanism underlies the CSWS in cases with early thalamic lesions. The detailed neurophysiological characterization of spontaneous EEG rhythms and the integration with the cortical excitability results using the SSVEPs protocol highlighted an important connection between the sleep spindles and CSWS: (a) Sleep states with no spindles do not have CSWS (Supporting Fig. 2C). (b) Spindles are preserved in the hemisphere with the thalamic lesion (Fig. 3B). (c) Brain areas with CSWS show a frequency-dependent increased excitability at spindle frequencies (Fig. 6A). (d) Patients that recovered from CSWS do not have a frequency-dependent excitability at spindle frequency (Fig. 6C).

The highlighted importance of sleep spindles in CSWS and results from the previous experimental research in the phenomena of augmenting response plasticity led us to propose the following model for the genesis of CSWS in our patients:

1. A predominantly dorsal thalamic lesion produces a neocortical area partially deafferented from thalamic inputs, while preserving the ventral thalamus.

2. The deafferented neocortex expresses a pathologically increased augmenting response.

3. In non-REM sleep, the presence of cortical spindles contributes to a local potentiation of the augmenting responses, leading to the generation of the continuous spiking typical of the CSWS.

The consistent age-dependent expression of the CSWS years after the thalamic lesion suggests that a developmental susceptibility factor underlies this transient major imbalance in the thalamus-cortical circuitry oscillations. The nature of this factor remains unknown, but its appearance is likely to involve a developmental change in gene expression which renders the 4- to 14-year-old children with the appropriate thalamic lesions susceptible to CSWS.

A limitation of the present study is that all epileptic foci in our group of patients were localized in the posterior cortex, whereas cases with CSWS associated with predominantly anterior cortex have been described. Whether our proposed model is also applicable to such cases remains to be determined.

A remaining open question is whether the proposed model is only relevant for thalamic lesions or it is also applicable to other etiologies. The characterization of the thalamic-cortical connection in CSWS associated with other etiologies could provide answer to this question. To the best of our knowledge, this is the first model proposed for CSWS spanning a single etiology, and even though it only represents around $14 \%$ of all cases [5], it is in our view particularly illuminating in the connection it allows with the physiological EEG rhythms throughout the wake-sleep cycle and in the restricted and anatomically well-localized single-hemisphere lesions. Because the proposed model is highly dependent on the overlap of the frequency-dependent increased excitability and the spindle frequency range, an interesting test of its applicability to other etiologies of CSWS would be the demonstration of a similar frequency-dependent neocortical hyperexcitability in such cases. We therefore propose that a more detailed study of the frequency dependence of the corticocortical excitability in CSWS of diverse etiologies might provide important information towards the construction of a general model of the condition and provide clues to the as-yet mysterious connection with the sleep stages and EEG rhythms.

Because of the retrospective exploratory character of the present study and the limitations of ethical considerations and patient cooperation, not all patients underwent the same investigations. We tried to make it transparent which kind of support each of the partial conclusions we reached was based on, but we recognize that this is a limitation that future prospective studies will need to address and overcome.

Supplementary data to this article can be found online at https://doi. org/10.1016/j.yebeh.2017.08.027.

\section{Potential conflicts of interest}

None of the authors has any conflict of interest to report. 


\section{Ethical publication statement}

We confirm that we have read the Journal's position on issues involved in ethical publication and affirm that this report is consistent with those guidelines.

\section{References}

[1] Tassinari C, Rubboli G, Volpi L, et al. Electrical status epilepticus during slow sleep (ESES or CSWS) including acquired epileptic aphasia (Landau-Klefener syndrome). In: Roger J, Bureau M, Dravet C, Genton P, Tassinari C, Wolf P, editors. Epileptic syndromes in infancy, childhood and adolescence. London: John Libbey; 2005. p. 295-314.

[2] Patry G, Lyagoubi S, Tassinari C. Subclinical electrical status epilepticus induced by sleep in children. Arch Neurol 1971;24:242-52.

[3] Engel J. A proposed diagnostic scheme for people with epileptic seizures and with epilepsy: report of the ILAE task force on classification and terminology. Epilepsia 2001;42:796-803.

[4] Galanopoulou A, Bojko A, Lado F, et al. The spectrum of neuropsychiatric abnormalities associated with electrical status epilepticus in sleep. Brain Dev 2000;22:279-95.

[5] Fernandez I, Takeoka M, Peters J, et al. Early thalamic lesions in patients with sleeppotentiated epileptiform activity. Neurology 2012;78:1721-7.

[6] Incorpora G, Pavone P, Smilari P, et al. Late primary unilateral thalamic hemorrhage in infancy: report of two cases. Neuropediatrics 1999;30:264-7.

[7] Monteiro J, Roulet-Perez E, Davidoff V, et al. Primary neonatal thalamic haemorrhage and epilepsy with continuous spike-wave during sleep: a longitudinal follow-up of a possible significant relation. Eur J Paediatr Neurol 2001;5:41-7.

[8] Kelemen A, Barsi P, Gyorsok Z, et al. Thalamic lesion and epilepsy with generalized seizures, ESES and spike-wave paroxysms-report of three cases. Seizure 2006;15: 454-8.

[9] Guzzeta F, Battaglia D, Veredice C, et al. Early thalamic injury associated with epilepsy and continuous spike-wave during slow sleep. Epilepsia 2005;46(6): 889-900.

[10] Losito E, Battaglia D, Chieffo D, et al. Sleep-potentiated epileptiform activity in early thalamic injuries: study in a large series (60 cases). Epilepsy Res 2015;109:90-9.

[11] Kersbergen K, Vries L, Leijten F, et al. Neonatal thalamic hemorrhage is strongly associated with electrical status epilepticus in slow wave sleep. Epilepsia 2013; 54(4):733-40.

[12] Larsson P, Wilson J, Eeg-Olofsson O. A new method for quantification and assessment of epileptiform activity in EEG with special reference to focal nocturnal epileptiform activity. Brain Topogr 2009;22(1):52-9.

[13] Clemens B, Ménes A. Sleep spindle asymmetry in epileptic patients. Clin Neurophysiol 2000;111:2155-9.

[14] Power B, Wilkes F, Hunter-Dickson M, et al. Validation of a protocol for manual segmentation of the thalamus on magnetic resonance imaging scans. Psychiatry Res Neuroimaging 2015;232(1):98-105.

[15] Byne W, Buchsbaum M, Kemether E, et al. Magnetic resonance imaging of the thalamus mediodorsal nucleus and pulvinar in schizophrenia and schizotypal personality disorder. Arch Gen Psychiatry 2001;58:133-40.
[16] Xie S, Chen L, Zuo N, et al. DiffusionKit: a light one-stop solution for diffusion MRI data analysis. J Neurosci Methods 2016;273:107-19.

[17] di Russo F, Pitzallis S, Aprile T, et al. Spatiotemporal analysis of the cortical sources of the steady-state visual evoked potential. Hum Brain Mapp 2007;28:323-34.

[18] Meigen T, Bach M. On the statistical significance of electrophysiological steady-state responses. Doc Ophthalmol 2000;98:207-32.

[19] Jones E. The thalamus. 2nd ed. Cambridge University Press; 2007.

[20] Fernandez I, Loddenkemper T, Peters J, et al. Electrical status epilepticus in sleep: clinical presentation and pathophysiology. Pediatr Neurol 2012;47:390-410.

[21] Trounce J, Dodd K, Fawer C, et al. Primary thalamic hemorrhage in the newborn: a new clinical entity. Lancet 1985;1:190-2.

[22] Roland E, Flodmark O, Hill A. Thalamic hemorrhage with intraventricular hemorrhage in the full-term newborn. Pediatrics 1990;85:737-42.

[23] Maquet P, Hirsch E, Metz-Lutz M, Motte J, et al. Regional cerebral glucose metabolism in children with deterioration of one or more cognitive functions and continuous spike-and-wave discharges during sleep. Brain 1995;118:1497-520.

[24] Piantoni G, Halgren E, Cash S. The contribution of thalamocortical core and matrix pathways to sleep spindles. Neural Plast 2016;2016:3024342.

[25] Timofeev I, Grenier F, Bazhenov M, et al. Origin of slow cortical oscillations in deafferented cortical slabs. Cereb Cortex 2000;10:1185-99.

[26] Hammond E, Wilder B, Ballinger W. Electrophysiological recordings in a patient with a discrete unilateral thalamic infarction. J Neurol Neurosurg Psychiatry 1982;45: 640-3.

[27] Matsumoto R, Kinoshita M, Taki J, et al. In vivo epileptogenicity of focal cortical dysplasia: a direct cortical paired stimulation study. Epilepsia 2005;46(11):1744-9.

[28] Lopes da Silva F, Mooibroek J, Van Rotterdam A. Relative contributions of intracortical and thalamo-cortical processes in the generation of alfa rhythms, revealed by partial coherence analysis. Electroencephalogr Clin Neurophysiol 1980; 50:449-56.

[29] Morison R, Dempsey E. Mechanism of thalamocortical augmentation and repetition. Am J Physiol 1943;138:297-308.

[30] Steriade M, Timofeev I. Short-term plasticity during intrathalamic augmenting responses in decorticated cats. J Neurosci 1997;17(10):3778-95 [Neurosc 1998; 18(16): 6425-6443].

[31] Steriade M, Nunez A, Amzica F. Intracellular analysis of the relations between the slow $(<1 \mathrm{~Hz})$ neocortical oscillation and other sleep rhythms of the electroencephalogram. J Neurosci 1993;13(8):3266-83.

[32] Timofeev I, Steriade M. Cellular mechanisms underlying intrathalamic augmenting responses of reticular and relay neurons. J Neurophysiol 1998;79:2716-29.

[33] Timfeev I, Grenier F, Bazhenov M, et al. Short- and medium-term plasticity associated with augmenting responses in cortical slabs and spindles in intact cortex of cats in vivo. J Physiol 2002;542(2):583-98.

[34] Kallay C, Mayor-Dubois C, Maeder-Ingvar M, et al. Reversible acquired epileptic frontal syndrome and CSWS suppression in a child with congenital hemiparesis treated by hemispherotomy. Eur J Paediatr Neurol 2009;13:430-8.

[35] Battaglia D, Veggiotti P, Lettori D, et al. Functional hemispherectomy in children with epilepsy and CSWS due to unilateral early brain epilepsy including thalamus: sudden recovery of CSWS. Epilepsy Res 2009;87:290-8. 